\title{
COMPLETE COINDUCTIVE THEORIES. II
}

\author{
A. H. LACHLAN
}

\begin{abstract}
Let $T$ be a complete theory over a relational language which has an axiomatization by $\exists \forall$-sentences. The properties of models of $T$ are studied. It is shown that existential formulas are stable. A theory of forking and independence based on Boolean combinations of existential formulas in $\exists \forall$-saturated models of $T$ is developed for which the independence relation is shown to satisfy a very strong triviality condition. It follows that $T$ is tree-decomposable in the sense of Baldwin and Shelah. It is also shown that if the language is finite, then $T$ has a prime model.
\end{abstract}

This paper is the second part of a work begun in [9] which will be referred to as Part I. The topic is coinductive complete theories over relational languages, where coinductive means that there is an axiomatization by $\exists \forall$-sentences. The section numbers follow consecutively from those in Part I. The introduction to Part I will also serve for Part II. So here we give only a brief outline of what follows.

In $\S 6$, the first section of Part II, it is shown that $\exists$-formulas are stable. This strengthens Theorem 2.1 which says that quantifier-free formulas are stable.

A formula is called a $\exists$ : $\forall$-formula if it is a Boolean combination of $\exists$ formulas. In $\S 7$, by working within $\exists \forall$-saturated models, we develop a theory of forking based on $\exists: \forall$-formulas. This is made possible by the stability of $\exists$-formulas and Corollary 3.9 which says that in a $\exists \forall$-saturated model the elementary type $\operatorname{Tp}(\bar{a})$ of a tuple is determined by its $\exists$-type $\exists$ - $\operatorname{tp}(\bar{a})$. It turns out that the notion of independence based on $\exists: \forall$-formulas is trivial in the sense that, for all subsets $A, C, D$ of a model and each element $b$,

$$
A \downarrow C(D) \Rightarrow[A \downarrow b(C \cup D) \vee C \downarrow b(A \cup D)] .
$$

In $\S 8$ we use the triviality of the independence relation to show that complete coinductive theories are tree-decomposable in the sense of Baldwin and Shelah $[3$, p. 253]. An equivalent statement is that any extension of the theory by unary predicates is stable. In particular, we see that complete coinductive theories are stable, a result we have been unable to obtain more directly.

Received by the editors November 2, 1988 and, in revised form, September 12, 1989.

1980 Mathematics Subject Classification (1985 Revision). Primary 03C99; Secondary 03C45.

The author acknowledges the support of the Government of Canada through NSERC Grant A3040 and is grateful to John Baldwin for many helpful comments on an earlier draft of the paper. 
In $\S 9$ it is shown that when the language is finite there is always a prime model, although prime models over sets in general do not exist. In $\S 10$ we list some conjectures which suggest directions for further study.

\section{Stability of EXISTENTIAL FORMUlas}

We have already seen that quantifier-free formulas are stable. Here we show that existential formulas are also stable.

6.1. Theorem. Let $T$ be a complete $\exists \forall$-theory over a relational language. Then all existential formulas are stable in $T$.

Proof. Without loss of generality assume that the language is countable. We first reduce to the case of an existential formula $\psi(x, \bar{y})$. This is accomplished by adapting the usual proof that, if $T$ is unstable, then some formula $\psi(x, \bar{y})$ is unstable. To this end suppose that some existential formula $\psi(\bar{x}, \bar{y})$ of $T$ is unstable, and choose such $\psi(\bar{x}, \bar{y})$ with $l(\bar{x})$ as small as possible. Towards a contradiction suppose that $l(\bar{x})=m+1>1$. Let $\kappa$ be an infinite cardinal such that $\kappa^{\aleph_{0}}=\kappa$. We construct a model $\mathscr{M}$ such that:

(1) For every $n<\omega, A \subseteq M$ of size $\leq \kappa$, and $\mathscr{N} \supseteq_{\forall} \mathscr{M}$, every $\exists \forall-n$-type over $A$ realized in $\mathscr{N}$ is also realized in $\mathscr{M}$.

(2) There exists $B \subseteq M$ of size $\kappa$ such that more than $\kappa \psi$ - $(m+1)$-types over $B$ are realized in $\mathscr{M}$.

Let $a_{i} \bar{b}_{i} \subseteq M\left(i<\kappa^{+}\right)$realize distinct $\psi-(m+1)$-types over $B$. By assumption, for each existential formula $\theta(x, \bar{z})$ the number of $\theta$-1-types over $B$ is $\leq \kappa$. Since the language is assumed countable, the number of $\exists$-1-types over $B$ is $\leq \kappa^{\aleph_{0}}=\kappa$. By thinning we can suppose that $\exists-\operatorname{tp}\left(a_{i} \mid B\right)=\exists-\operatorname{tp}\left(a_{j} \mid B\right)$ $\left(i<j<\kappa^{+}\right)$. From Corollary $3.9 \operatorname{Tp}\left(a_{i} \mid B\right)=\operatorname{Tp}\left(a_{j} \mid B\right) \quad\left(i<j<\kappa^{+}\right)$. From (1) there exist $\bar{b}_{i}^{\prime} \in M\left(i<\kappa^{+}\right)$such that $\exists \forall-\operatorname{tp}\left(a_{0} \bar{b}_{i}^{\prime} \mid B\right)=\exists \forall-\operatorname{tp}\left(a_{i} \bar{b}_{i} \mid B\right)$ $\left(i<\kappa^{+}\right)$. Let $\psi^{\prime}\left(\bar{x}^{\prime}, \bar{y}^{\prime}\right)$ denote $\psi\left(y_{0}, x_{1}, \ldots, x_{m}, y_{1}, \ldots, y_{l(\bar{y})}\right)$. Then the $\bar{b}_{i}^{\prime}$ realize distinct $\psi^{\prime}$-m-types over $B \cup\left\{a_{0}\right\}$. Thus, $\psi^{\prime}\left(\bar{x}^{\prime}, \bar{y}^{\prime}\right)$ is unstable. This contradicts the choice of $\psi(\bar{x}, \bar{y})$. We conclude that $l(\bar{x})=1$.

Using compactness and Ramsey's theorem we can find countable models $\mathscr{M}$, $\mathscr{M}^{-}$of $T$ with $\mathscr{M}^{-} \subseteq_{\forall} \mathscr{M}$, a quantifier-free formula $\phi(x, \bar{y}, \bar{z})$ over $M^{-}$, a countably infinite set $I \cup J$ linearly ordered by $<$, and $a_{i}, \bar{b}_{j}, \bar{c}_{i j} \quad(i \in I$, $j \in J, i<j$ ) satisfying the following conditions:

R1. Each of $I$ and $J$ is dense in $I \cup J$ and there are no end points.

R2. For all $i \in I$ and $j \in J$

$$
\left[\mathscr{M} \vDash \psi\left(a_{i}, \bar{b}_{j}\right)\right] \Leftrightarrow i<j,
$$

where $\psi(x, \bar{y})$ denotes $\exists \bar{z} \phi(x, \bar{y}, \bar{z})$, and $i<j$ implies

$$
\mathscr{M} \vDash \phi\left(a_{i}, \bar{b}_{j}, \bar{c}_{i j}\right) \text {. }
$$

R3. Let $A=\left\{a_{i}: i \in I\right\}, B=\bigcup\left\{\bar{b}_{j}: j \in J\right\}$, and $C=\bigcup\left\{\bar{c}_{i j}: i \in I, j \in J\right.$, $i<j\}$. Then $M^{-}=M \backslash(A \cup B \cup C)$. 
R4. If $i_{0}<j_{0}<\cdots<i_{m}<j_{m}$ and $i_{0}^{\prime}<j_{0}^{\prime}<\cdots<i_{m}^{\prime}<j_{m}^{\prime}$, where the $i$ 's and $i^{\prime}$ 's are in $I$ and the $j$ 's and $j^{\prime}$ 's are in $J$, then the tuple

$$
a_{i_{0}} \cdots a_{i_{m}} \bar{b}_{j_{0}} \cdots \bar{b}_{j_{m}} \bar{c}_{i_{0} j_{0}} \cdots \bar{c}_{i_{0} j_{m}} \bar{c}_{i_{1} j_{1}} \cdots \bar{c}_{i_{m} j_{m}}
$$

realizes the same type over $M^{-}$as

$$
a_{i_{0}^{\prime}} \cdots a_{i_{m}^{\prime}} \bar{b}_{j_{0}^{\prime}} \cdots \bar{b}_{j_{m}^{\prime}}{\overline{i_{0}^{\prime} j_{0}^{\prime}}} \cdots \bar{c}_{i_{0}^{\prime} j_{m}^{\prime}}{\overline{i_{i}^{\prime} j_{1}^{\prime}}}_{1} \cdots \bar{c}_{i_{m}^{\prime} j_{m}^{\prime}} \text {. }
$$

Property R4 will be referred to as "the indiscernibility" below.

The whole configuration described above is to be chosen so as to ensure that $l(\bar{y})$ is as small as possible.

By choosing a different formula $\phi(x, \bar{y}, \bar{z})$ if necessary we can suppose that $a_{i} \bar{b}_{j} \cap \bar{c}_{i j}=\varnothing \quad(i \in I, j \in J, i<j)$. By the indiscernibility this means that $a_{i} \bar{b}_{j} \cap \bar{c}_{k l}=\varnothing \quad(i, k \in I, j, l \in J, k<l)$. Also, at the cost of adjoining some elements to $M^{-}$if necessary, we can suppose that $\bar{b}_{j} \cap \bar{b}_{l}=\varnothing(j, l \in J$, $j \neq l)$ and $\bar{c}_{i j} \cap \bar{c}_{k l}=\varnothing(i, k \in I, j, l \in J, i \neq k$, or $j \neq l)$. From the indiscernibility it follows that $a_{i} \notin \bar{b}_{j} \quad(i \in I, j \in J)$.

We say that $a \in M$ depends on $i \in I$ if either $a=a_{i}$ or $a \in \bar{c}_{i j}$ for some $j \in J, j>i$. Similarly, $a \in M$ depends on $j \in J$ if either $a \in \bar{b}_{j}$ or $a \in \bar{c}_{i j}$ for some $i \in I, i<j$. Clearly, $a \in M$ depends on at most one $i \in I$ and at most one $j \in J$, and if on both then $i<j$. The elements $k \in I \cup J$ such that some $a \in \bar{d}$ depends on $k$ are called the coordinates of $\bar{d}$.

Let $i, i^{\prime} \in I$ and $\bar{d} \in M$. Suppose that

$$
(\forall j \in J)\left[\bar{c}_{i j} \cap \bar{d} \neq \varnothing \Rightarrow i^{\prime}<j\right] .
$$

The tuple $\bar{d}^{\prime}$ obtained from $\bar{d}$ by moving $i$ to $i^{\prime}$ is defined entry by entry: if the entry $d_{h}$ of $\bar{d}$ does not depend on $i$, then $d_{h}^{\prime}$, the corresponding entry of $\bar{d}^{\prime}$, is $d_{h}$; if $d_{h}=a_{i}$, then $d_{h}^{\prime}=a_{i^{\prime}}$; if $d_{h}$ is an entry of $\bar{c}_{i j}$, then $d_{h}^{\prime}$ is the corresponding entry of $\bar{c}_{i^{\prime} j}$. Let $j, j^{\prime} \in J$ and suppose that

$$
(\forall i \in I)\left[\bar{c}_{i j} \cap \bar{d} \neq \varnothing \Rightarrow i<j^{\prime}\right] .
$$

The tuple $\bar{d}^{\prime}$ obtained from $\bar{d}$ by moving $j$ to $j^{\prime}$ is defined in the obvious way.

Claim 1. Let $\theta(\bar{u})$ be a quantifier-free formula, $\bar{d} \in M, i, i^{\prime} \in I$, and $i^{\prime}$ not be a coordinate of $\bar{d}$. If $\bar{d}^{\prime}$ is obtained from $\bar{d}$ by moving $i$ to $i^{\prime}$, then $\mathscr{M} \vDash \theta(\bar{d}) \leftrightarrow \theta\left(\bar{d}^{\prime}\right)$. Similarly with $j, j^{\prime}, J$ replacing $i, i^{\prime}, I$ respectively.

Proof of Claim 1. Consider the part about $i$ and $i^{\prime}$; the part about $j$ and $j^{\prime}$ is similar. Towards a contradiction suppose that $\mathscr{M} \vDash \theta(\bar{d}) \& \neg \theta\left(\bar{d}^{\prime}\right)$. By the indiscernibility some coordinate of $\bar{d}$ lies between $i$ and $i^{\prime}$. By interpolating other elements of $I$ between $i$ and $i^{\prime}$ we can suppose that there is a unique coordinate $k$ of $\bar{d}$ which lies between $i$ and $i^{\prime}$. There are two cases: $i<k<i^{\prime}$ 
and $i^{\prime}<k<i$. We treat only the first case; the second case is similar. Thus, suppose $i<k<i^{\prime}$. Reordering $\bar{u}$ if necessary write $\bar{d}=\bar{e} \bar{f}$, where $\bar{e}$ enumerates the elements of $\bar{d}$ which depend on $i$. Choose $i_{m}, k_{m} \in I \cup J$ $(m<\omega)$ such that $i_{m} \in I, k_{m} \in I$ iff $k \in I$, and $i<i_{0}<k_{0}<i_{1}<k_{1}<$ $\cdots<k$. Notice that $k$ is not one of the coordinates of $\bar{e}$. Let $\bar{e}_{m}$ be obtained from $\bar{e}$ by moving $i$ to $i_{m}$, and $\bar{f}_{n}$ be obtained from $\bar{f}$ by moving $k$ to $k_{n}$. From the indiscernibility

$$
m \leq n \Leftrightarrow\left[\mathscr{M} \vDash \theta\left(\bar{e}_{m} \bar{f}_{n}\right)\right] \quad(m, n<\omega) .
$$

Thus, $\theta\left(\bar{x}^{\prime}, \bar{y}^{\prime}\right)$ is unstable, contradicting Theorem 2.1 .

Claim 2. (i) Let $i, i^{\prime} \in I, i^{\prime}<i$, and neither $i$ nor $i^{\prime}$ be a coordinate of $\bar{b}$. Let $a^{\prime}$ be obtained from $a$ by moving $i$ to $i^{\prime}$. Then $\mathscr{M} \vDash \psi(a, \bar{b}) \rightarrow$ $\psi\left(a^{\prime}, \bar{b}\right)$. Moreover, if there is no $J$-coordinate of $\bar{b}$ between $i^{\prime}$ and $i$, then $\mathscr{M} \vDash \psi(a, \bar{b}) \leftrightarrow \psi\left(a^{\prime}, \bar{b}\right)$.

(ii) Similarly with $j, j^{\prime}, J, I$ for $i, i^{\prime}, I, J$ respectively, with the order reversed.

Proof of Claim 2. It is implicit in the statement of the claim that $i$ is a coordinate of $a$ and hence that $i^{\prime}$ is not. Assume that $i, i^{\prime}$, and $\bar{b}$ satisfy the hypothesis of (i) and that $\mathscr{M} \vDash \psi(a, \bar{b})$. There exists $\bar{c}$ such that $\mathscr{M} \vDash \phi(a, \bar{b}, \bar{c})$. By applying Claim 1 if necessary we can suppose that $i^{\prime}$ is not a coordinate of $\bar{c}$. Let $\bar{c}^{\prime}$ be obtained by moving $i$ to $i^{\prime}$. Then $\mathscr{M} \vDash \phi\left(a^{\prime}, \bar{b}, \bar{c}^{\prime}\right)$ by Claim 1 , whence $\mathscr{M} \vDash \psi\left(a^{\prime}, \bar{b}\right)$. Now suppose that there is no $J$-coordinate of $\bar{b}$ between $i^{\prime}$ and $i$, and that $\mathscr{M} \vDash \psi\left(a^{\prime}, \bar{b}\right)$. There exists $\bar{c}^{\prime}$ such that $\mathscr{M} \vDash \phi\left(a^{\prime}, \bar{b}, \bar{c}^{\prime}\right)$. From Claim 1 we can suppose that $\bar{c}^{\prime}$, has no $J$-coordinates between $i^{\prime}$ and $i$, because any such coordinates can be moved to the right of $i$ without $a^{\prime}$ or $\bar{b}$ being affected. Since there are no $J$-coordinates of $\bar{c}^{\prime}$ between $i^{\prime}$ and $i$ there exists $\bar{c}$ obtained from $\bar{c}^{\prime}$ by moving $i^{\prime}$ to $i$. Then $\mathscr{M} \vDash \phi(a, \bar{b}, \bar{c})$ by Claim 1 , whence $\mathscr{M} \vDash \psi(a, \bar{b})$.

Our strategy in the rest of the proof of the theorem is to investigate the solution sets of formulas of the form $\psi(x, \bar{b})$. Since a considerable amount of detail seems unavoidable, before going further, we indicate the kind of contradiction we are aiming for. Let $j, k \in J$ and suppose that $A^{*}=\left\{a_{i}: j<i<k\right\}$ is a definable subset of $\mathscr{M}$. Let $l(\bar{y})=m$. Consider the family $\mathscr{X}$ of all subsets of $A^{*}$ of the form $X(\bar{b})=\left\{a \in A^{*}: \mathscr{M} \vDash \psi(a, \bar{b})\right\}$, where $\bar{b}$ runs through $M^{m}$. From (i) of Claim 2, ignoring the at most $m$ elements $a_{i}$ such that $i$ is a coordinate of $\bar{b}, X(\bar{b})$ is closed downwards, i.e., if $h, i \in I, j<h<i<k$, and $a_{i} \in X(\bar{b})$, then $a_{h} \in X(\bar{b})$ also. Thus, if $\left|A^{*} \backslash X(\bar{b})\right|>m$, then there exists $j^{\prime} \in J$ such that $j<j^{\prime}<k$ and $X(\bar{b}) \subset X\left(\bar{b}_{j^{\prime}}\right)$. Thus, we have

$$
\mathscr{M} \vDash \forall \bar{y} \exists \bar{z}\left[\left|A^{*} \backslash X(\bar{y})\right|>m \Rightarrow\left[\left|A^{*} \backslash X(\bar{z})\right|>m \& X(\bar{y}) \subset X(\bar{z})\right]\right] .
$$

Let $\sigma$ be a first-order sentence expressing this last property, where we quantify existentially to eliminate the parameters needed to define $A^{*}$. Then $\vdash_{T} \sigma$, and 
$\sigma$ has only infinite models. Since $T$ is $\exists \forall$, there is a consistent $\exists \forall$-sentence $\tau$ which implies $\sigma$. But $\tau$ has a finite model as does every $\exists \forall$-sentence over a relational language. This is the desired contradiction. The same idea, referred to as the basic argument below, can be applied whenever a definable set can be found to play the role of $A^{*}$.

We now return to our study of the solution sets of the formulas $\psi(x, \bar{b})$. We need to consider particular entries of the tuples $\bar{b}_{j}$ and $\bar{c}_{i j}$. Because we already have subscripts from $I$ and $J$, the $n$th entries in $\bar{b}_{j}$ and $\bar{c}_{i j}$ will be denoted $b_{j}^{n}$ and $c_{i j}^{n}$ respectively. We begin with the simplest case in which $\bar{b}$ is $\bar{b}_{j}$ for some $j \in J$. The next two claims follow easily from Claim 2 .

Claim 3. If $h, j, k \in J$ are distinct and $1 \leq n \leq l(\bar{b})$, then

$$
\mathscr{M} \vDash \psi\left(b_{h}^{n}, \bar{b}_{j}\right) \leftrightarrow \psi\left(b_{k}^{n}, \bar{b}_{j}\right) .
$$

Claim 4. Fix $n, 1 \leq n \leq l\left(\bar{c}_{i j}\right)$. Let $i$ run through $I$ and $j, k$ run through $J$. One of the following three formulas holds universally:

$$
\begin{aligned}
& {[i<k \& j \neq k] \Rightarrow\left[\mathscr{M} \vDash \psi\left(c_{i k}^{n}, \bar{b}_{j}\right)\right],} \\
& {[i<k \& j \neq k] \Rightarrow\left[\mathscr{M} \vDash \neg \psi\left(c_{i k}^{n}, \bar{b}_{j}\right)\right],} \\
& {[i<k \& j \neq k] \Rightarrow\left[\left[\mathscr{M} \vDash \psi\left(c_{i k}^{n}, \bar{b}_{j}\right)\right] \Leftrightarrow i<j\right] .}
\end{aligned}
$$

The next claim is one of the key points in our analysis.

Claim 5. There exist $Y, Y_{0}, Y_{1} \subseteq\left\{n: 1 \leq n \leq l\left(\bar{c}_{i j}\right)\right\}$ such that $Y \cap Y_{0}=\varnothing$, $Y \subseteq Y_{1}$, and for all $j \in J$ the intersection of the solution set of $\psi\left(\chi, \bar{b}_{j}\right)$ with $M \backslash\left(M^{-} \cup B\right)$ is the union of the sets:

$$
\begin{gathered}
\left\{a_{i}: i \in I, i<j\right\}, \\
\left\{c_{i k}^{n}: i \in I, k \in J, i<j, k, j \neq k, n \in Y\right\}, \\
\left\{c_{i k}^{n}: i \in I, k \in J, i<k, j \neq k, n \in Y_{0}\right\}, \\
\left\{c_{i j}^{n}: i \in I, i<j, n \in Y_{1}\right\} .
\end{gathered}
$$

Proof of Claim 5. Except for $Y \subseteq Y_{1}$ the conclusion follows immediately from Claims 2 and 4. Fix $j_{0}, k_{0}, k_{1}, j_{1}$ all in $J$ and increasing. From the limited conclusion just mentioned we see that the solution set of the formula

$$
\neg \psi\left(x, \bar{b}_{j_{0}}\right) \& \psi\left(x, \bar{b}_{k_{0}}\right) \& \psi\left(x, \bar{b}_{k_{1}}\right) \& \neg \psi\left(x, \bar{b}_{j_{1}}\right)
$$

is

$$
\left\{c_{i j_{1}}^{n}: i \in I, j_{0}<i<k_{0}, n \in Y \backslash Y_{1}\right\} .
$$

Choose a minimal nonempty set $Z \subseteq Y \backslash Y_{1}$, if any, such that

$$
\left\{c_{i j_{1}}^{n}: i \in I, j_{0}<i<k_{0}, n \in Z\right\}
$$

is definable. This set can play the role of $A^{*}$ in the basic argument. Hence no such $Z$ exists, which implies that $Y \subseteq Y_{1}$. 
The next claim follows immediately from Claims 3 and 5 taking $X=Y$, $X_{0}=Y_{0} \backslash Y_{1}$, and $X_{1}=Y_{1} \backslash\left(Y \cup Y_{0}\right)$.

Claim 6. There exist pairwise disjoint set $X, X_{0}, X_{1} \subseteq\left\{n: 1 \leq n \leq l\left(\bar{c}_{i j}\right)\right\}$ such that for all $j_{0}, j_{1} \in J$ with $j_{0}<j_{1}$ the solution set of the formula

$$
\neg \psi\left(x, \bar{b}_{j_{0}}\right) \& \psi\left(x, \bar{b}_{j_{1}}\right) \& x \notin \bar{b}_{j_{0}} \bar{b}_{j_{1}}
$$

is the union of the sets:

$$
\begin{gathered}
\left\{a_{i}: i \in I, j_{0}<i<j_{1}\right\}, \\
\left\{c_{i j}^{n}: i \in I, j \in J, j_{0}<i<j_{1}, i<j, n \in X\right\}, \\
\left\{c_{i j_{0}}^{n}: i \in I, i<j_{0}, n \in X_{0}\right\}, \\
\left\{c_{i j_{1}}^{n}: i \in I, i<j_{i}, n \in X_{1}\right\} .
\end{gathered}
$$

For the rest of the proof let $j_{0}, k_{0}, k_{1}, j_{1} \in J$ be fixed such that $j_{0}<k_{0}<$ $k_{1}<j_{1}$. Let $B^{*}$ denote the solution set of the formula

$$
\neg \psi\left(x, \bar{b}_{j_{0}}\right) \& \neg \psi\left(x, \bar{b}_{k_{0}}\right) \& \psi\left(x, \bar{b}_{k_{1}}\right) \& \psi\left(x, \bar{b}_{j_{1}}\right) .
$$

From Claim 6

$$
B^{*}=\left\{a_{i}, c_{i j}^{n}: i \in I, k_{0}<i<k_{1}, i<j, n \in X\right\} .
$$

Let $D$ denote the set of all $\bar{b} \in M^{m}$ such that

$$
\begin{aligned}
\mathscr{M} \vDash \forall x\left[\left(\neg \psi\left(x, \bar{b}_{j_{0}}\right) \& \psi\left(x, \bar{b}_{j_{1}}\right)\right) \rightarrow\right. & {\left[\left(\psi\left(x, \bar{b}_{k_{0}}\right) \rightarrow \psi(x, \bar{b})\right)\right.} \\
\left.\&\left(\psi(x, \bar{b}) \rightarrow \psi\left(x, \bar{b}_{k_{1}}\right)\right]\right] &
\end{aligned}
$$

From now on we are mainly concerned with the sets

$$
F(\bar{b})=B^{*} \cap\{a \in M: \mathscr{M} \vDash \psi(a, \bar{b})\} \quad(\bar{b} \in D) .
$$

Note that $\bar{b}_{j} \in D$ if and only if $k_{0} \leq j \leq k_{1}$, and that an an element of $B^{*}$ is in $F\left(\bar{b}_{j}\right)$ if and only if its $I$-coordinate is less than $j$.

Claim 7. Let $\bar{b} \in D$. There exists $j \in J$ such that $k_{0} \leq j \leq k_{1}$ and $j$ is a coordinate of each entry of $\bar{b}$. Further, if $a \in B^{*}$ has $I$-coordinate $i$ and $i$ is not a coordinate of $\bar{b}$, then $a \in F(\bar{b})$ if and only if $i<j$.

Proof of Claim 7. Fix $\bar{b} \in D$. Consider the intervals into which the coordinates of $\bar{b}$ and $k_{0}, k_{1}$ partition the rest of the set $\left\{x \in I: j_{0}<x<j_{1}\right\}$. Consider an $i$ in one of these intervals. Whether or not $a_{i} \in F(\bar{b})$ does not depend on the particular $i$, but only on the interval in which it falls. Since $\bar{b} \in D$, $a_{i} \in F(\bar{b})$ if $i<k_{0}$, and $a_{i} \notin F(\bar{b})$ if $k_{1}<i$. Consider the leftmost interval for $i$ in which $a_{i} \notin F(\bar{b})$. From Claim 2 this interval is bounded on the left by a $J$-coordinate $j$ of $\bar{b}$. Clearly, $k_{0} \leq j$ and $j \leq k_{1}$. If there is an entry of $\bar{b}$ of which $j$ is not a coordinate, then the formula $\psi^{\prime}\left(x, \bar{y}^{\prime}\right)$, obtained from 
$\psi(x, \bar{y})$ by substituting only such entries of $\bar{b}$ for the corresponding variables in $\bar{y}$, is an existential formula with the order property which contradicts the choice of $\phi(x, \bar{y}, \bar{z})$ since $l\left(\bar{y}^{\prime}\right)<l(\bar{y})$. This gives the first part of the claim.

The second part of the claim is obtained as follows. Let $n \in X, i \in I$, $k \in J, i<k, k_{0}<i<j$, and $i$ not be a coordinate of $\bar{b}$. Choose $i^{\prime} \in I$ such that $j_{0}<i^{\prime}<k_{0}$ and $i^{\prime}$ is not a coordinate of $\bar{b}$. Since $\bar{b} \in D, a_{i^{\prime}}$ and $c_{i^{\prime} k}^{n}$ are both in $F(\bar{b})$. Clearly, $\bar{b}$ has no $J$-coordinate between $i^{\prime}$ and $i$. By Claim 2(i), $a_{i}$ and $c_{i k}^{n}$ are both in $F(\bar{b})$ also. Now make the same assumptions as before but with $j<i<k_{1}$ instead of $k_{0}<i<j$. Choose $i^{\prime} \in I$ and $k^{\prime} \in J$ such that $k_{1}<i^{\prime}<k^{\prime}<j_{1}$. Since $\bar{b} \in D$, neither $a_{i^{\prime}}$ nor $c_{i^{\prime} k}^{n}$ is in $F(\bar{b})$. Since $j$ is a coordinate of each entry of $\bar{b}, \bar{b}$ has no other $J$-coordinate and its $I$-coordinates are all $<j$. From Claim 2, moving $i^{\prime}$ to $i$ and then $k^{\prime}$ to $k$, we see that $a_{i} \notin F(\bar{b})$ and $c_{i k}^{n} \notin F(\bar{b})$. This completes the proof of the claim.

Claim 8. Let $n \in X, \bar{b} \in D, j \in J$ be the coordinate of $\bar{b}$ found in Claim 7, and $i>k_{0}$ be an $I$-coordinate of $\bar{b}$. Then either

$$
(\forall k \in J)\left[i<k \neq j \Rightarrow c_{i k}^{n} \in F(\bar{b})\right] \quad \text { or } \quad(\forall k \in J)\left[i<k \neq j \Rightarrow c_{i k}^{n} \notin F(\bar{b})\right] .
$$

Proof of Claim 8. From Claim 7 recall that $j \in J, k_{0} \leq j \leq k_{1}$, and $j$ is a coordinate of each entry of $\bar{b}$. Towards a contradiction suppose that the conclusion of the lemma fails. By Claim 2(ii) there exist $l_{0}, l_{1} \in I$ such that $i \leq l_{0}<l_{1}<j, l_{1}$ is a coordinate of $\bar{b}$, and

$$
\left(\forall k, k^{\prime} \in J\right)\left[l_{0}<k<l_{1}<k^{\prime}<j \Rightarrow\left[c_{i k}^{n} \in F(\bar{b}) \Leftrightarrow c_{i k^{\prime}}^{n} \notin F(\bar{b})\right]\right] .
$$

The formula $\psi^{\prime}\left(x, \bar{y}^{\prime}\right)$, obtained from $\psi(x, \bar{y})$ by substituting the entries of $\bar{b}$ of which $l_{1}$ is not a coordinate for the corresponding entries of $\bar{y}$, has the order property because it can be used to "cut" a suitably chosen sequence of elements $c_{i k_{h}}^{n}(h=0,1, \ldots)$ at any point. Since an entry of $\bar{b}$ which has $i$ as a coordinate cannot also have $l_{1}$ as a coordinate, $l\left(\bar{y}^{\prime}\right)<l(\bar{y})$ contrary to the choice of $\phi(x, \bar{y}, \bar{z})$.

In Claims 7 and 8 it is clear that for each $\bar{b} \in D$ there exists $j(\bar{b}) \in J$, the unique $J$-coordinate of $\bar{b}$, and

$$
U, V \subseteq\{i \in I: i \text { is a coordinate of } \bar{b}\} \times\left\{n: 1 \leq n \leq l\left(\bar{c}_{i j}\right)\right\}
$$

such that $F(\bar{b}) \subseteq F\left(\bar{b}_{j(\bar{b})}\right)$ and $F\left(\bar{b}_{j(\bar{b})}\right) \backslash F(\bar{b})$ is

$$
\left\{c_{i j(\bar{b})}^{n}:(i, n) \in U\right\} \cup\left\{c_{i k}^{n}:(i, n) \in V, k \in J, i<k \neq j(\bar{b})\right\} .
$$

Note that there exists $s<\omega$ such that $|U|+|V|<s$ for all $\bar{b} \in D$.

We now adapt the basic argument to $B^{*}$ as follows. A sentence true in $\mathscr{M}$ with only infinite models is

$$
(\forall \bar{y} \in D)(\exists \bar{z} \in D)\left[j(\bar{y})<k_{1} \rightarrow\left[j(\bar{z})<k_{1} \& F(\bar{y}) \subset F(\bar{z})\right]\right] .
$$


Since $B^{*}$ and $D$ are definable, to see that this sentence is first-order it is enough to show that $j(\bar{y})<k_{1}$ is first-order. But from the form of $F\left(\bar{b}_{j(\bar{b})}\right) \backslash F(\bar{b})$ found above it is clear that $j(\bar{y})<k_{1}$ is equivalent to

$$
\exists \bar{z}_{1} \cdots \exists \bar{z}_{s}\left[\bar{z}_{1}, \ldots, \bar{z}_{s} \in D \& F(\bar{y}) \subset F\left(\bar{z}_{1}\right) \subset \cdots \subset F\left(\bar{z}_{s}\right)\right] .
$$

This completes the proof of the theorem.

\section{FORKING IN MODELS OF COMPLETE $\exists \forall$-THOERIES}

We wish to develop stability theory for complete $\exists \forall$-theories over relational languages. Since we do not know whether all formulas are stable we make do with knowing that existential formulas are stable. This is possible because by Corollary 3.9 in $\exists \forall$-saturated models the elementary type of a tuple is determined by its existential type, and by Lemma 3.1 every model has a $\forall$-extension which is $\exists \forall$-saturated. In this section we shall often deal with formulas which are Boolean combinations of existential formulas; such formulas will be called $\exists: \forall$-formulas. The stability of such formulas follows immediately from the stability of existential formulas.

We now sketch some necessary background material. A useful reference here is the paper [5] of Harnik and Harrington. For the time being let $T$ be an arbitrary first-order theory, $\mathscr{M} \vDash T, B \subseteq M$, and $\phi(\bar{x}, \bar{y})$ be a formula with $l(\bar{x})=m$ and without parameters. By instances of $\phi(\bar{x}, \bar{y})$ we mean instances obtained by substituting either parameters or variables not appearing in $\bar{x}$, for the variables $\bar{y}$. A formula $\theta(\bar{x}, \bar{z}, \bar{d})$ is said to be based on $\phi(\bar{x}, \bar{y})$ if it is a Boolean combination of instances of $\phi(\bar{x}, \bar{y})$. Let $\Psi(\bar{x})$ be an infinite set of formulas over $B$. We define $\phi$-rank and $\phi$-multiplicity by

$$
\phi-\operatorname{Rk}(\Psi(\bar{x}))=R^{m}\left(\Psi(\bar{x}), \phi, \aleph_{0}\right), \quad \phi-\operatorname{Mt}(\Psi(\bar{x}))=\operatorname{Mlt}\left(\Psi(\bar{x}), \phi, \aleph_{0}\right) .
$$

where the notation on the right is from [14, Chapter 2, $\S 1]$. We will write $\phi$-(Rk, Mt $)(\Psi(\bar{x}))$ for $(\phi-\operatorname{Rk}(\Psi(\bar{x})), \phi-\operatorname{Mt}(\Psi(\bar{x})))$. If $\Psi(\bar{x})=\{\psi(\bar{x})\}$ is a singleton, then we write $\phi-\operatorname{Rk}(\psi(\bar{x})), \phi-\operatorname{Mt}(\psi(\bar{x}))$ for $\phi-\operatorname{Rk}(\Psi(\bar{x})), \phi-\operatorname{Mt}(\Psi(\bar{x}))$. We will assume that the reader is familiar with the properties of this rank which can be found in Shelah [14, Chapter 2].

Let $\theta\left(\bar{x}, \bar{c}^{\prime}\right)$ be a formula over $M$. A conjugate of $\theta(\bar{x}, \bar{c})$ over $B$ is a formula of the form $\theta\left(\bar{x}, \bar{c}^{\prime}\right)$ such that $\operatorname{Tp}\left(\bar{c}^{\prime} \mid B\right)=\operatorname{Tp}(\bar{c} \mid B)$.

We write $\phi \perp \psi$ as an abbreviation for $(\phi \& \neg \psi) \vee(\psi \& \neg \phi)$, the symmetric difference of $\phi$ and $\psi$.

A useful property of $\phi$-rank is

7.1. Lemma. With the above notation, let $\phi(\bar{x}, \bar{y})$ be stable, $\theta(\bar{x}, \bar{c})$ be based on $\phi(\bar{x}, \bar{y}), \bar{c} \in M$, and $\phi-\operatorname{Rk}(\Psi(\bar{x}) \cup\{\theta(\bar{x}, \bar{c})\})=r \geq 0$. In some elementary extension of $\mathscr{M}$ there exists a positive Boolean combination $\chi(\bar{x}, \bar{d})$ of conjugates of $\theta(\bar{x}, \bar{c})$ over $B$ such that

(1) $\phi$ - $(\mathrm{Rk}, \mathrm{Mt})(\Psi(\bar{x}) \cup\{\theta(\bar{x}, \bar{c}) \perp \chi(\bar{x}, \bar{d})\})<r$. 
(2) If $\bar{d}_{0}, \bar{d}_{1}$ are tuples realizing $\operatorname{Tp}(\bar{d} \mid B)$ in an elementary extension of $\mathscr{M}$, then either $\chi\left(\bar{x}, \bar{d}_{0}\right)$ and $\chi\left(\bar{x}, \bar{d}_{1}\right)$ are equivalent in $\Psi(\bar{x})$ or

$$
\phi-\operatorname{Rk}\left(\Psi(\bar{x}) \cup\left\{\chi\left(\bar{x}, \bar{d}_{0}\right) \perp \chi\left(\bar{x}, \bar{d}_{1}\right)\right\}\right)=r .
$$

This is an application of Theorem 9.3 of [5]. If $\chi(\bar{x}, \bar{d})$ satisfies (2) we say that $\chi(\bar{x}, \bar{d})$ is $\phi$-normal in $\Psi(\bar{x})$.

Another result we need relates the ranks obtained from different formulas.

7.2. Lemma. With the above notation, let $\phi^{\prime}\left(\bar{x}, \bar{y}^{\prime}\right)$ be a stable formula without parameters such that

$$
\mathscr{M} \vDash \forall \bar{y} \exists \bar{y}^{\prime} \forall \bar{x}\left[\phi(\bar{x}, \bar{y}) \leftrightarrow \phi^{\prime}\left(\bar{x}, \bar{y}^{\prime}\right)\right],
$$

and let $\Psi(\bar{x})$ be a complete elementary type over $B$. Let $\theta(\bar{x})$ be a formula over $M$ based on $\phi^{\prime}\left(\bar{x}, \bar{y}^{\prime}\right)$. Then

$$
\phi-\operatorname{Rk}(\Psi(\bar{x}) \cup\{\theta(\bar{x})\})<\phi-\operatorname{Rk}(\Psi(\bar{x})) \Rightarrow \phi^{\prime}-\operatorname{Rk}(\Psi(\bar{x}) \cup\{\theta(\bar{x})\})<\phi^{\prime}-\operatorname{Rk}(\Psi(\bar{x})) \text {. }
$$

Proof. We prove the contrapositive. Let

$$
r^{\prime}=\phi^{\prime}-\mathbf{R} \mathbf{k}(\Psi(\bar{x}) \cup\{\theta(\bar{x})\})=\phi^{\prime}-\mathbf{R} \mathbf{k}(\Psi(\bar{x})) .
$$

Applying 7.1 to $\theta(\bar{x})$ and $\phi^{\prime}(\bar{x}, \bar{y})$, we obtain in some elementary extension a formula $\chi(\bar{x})$ which is a positive Boolean combination of conjugates of $\theta(\bar{x})$ over $B$ such that $\phi^{\prime}-\operatorname{Rk}(\Psi(\bar{x}) \cup\{\theta(\bar{x}) \perp \chi(\bar{x})\})<r^{\prime}$. Of course, $\chi(\bar{x})$ also has the property that it is $\phi^{\prime}$-normal in $\Psi(\bar{x})$. Since $\phi^{\prime}-\operatorname{Rk}(\Psi(\bar{x}) \& \chi(\bar{x}))=$ $\phi^{\prime}-\operatorname{Rk}(\Psi(\bar{x}))$, there exist $m<\omega$ and conjugates $\chi_{i}(\bar{x}) \quad(i<m)$ of $\chi(\bar{x})$ over $B$ such that for every conjugate $\chi^{\prime}(\bar{x})$ of $\chi(\bar{x})$ in an elementary extension of $\mathscr{M}$ there exists $i<m$ such that $\Lambda \Psi(\bar{x})$ implies $\chi^{\prime}(\bar{x}) \leftrightarrow \chi_{i}(\bar{x})$ is valid. Since $\Psi(\bar{x})$ is a complete elementary type over $B$, the formulas $\chi_{i}(\bar{x}) \quad(i<m)$ cover $\Psi(\bar{x})$. Since each $\chi_{i}(\bar{x})$ is a positive Boolean combination of conjugates of $\theta(\bar{x})$ over $B, \Psi(\bar{x})$ is covered by a finite number of conjugates of $\theta(\bar{x})$ over $B$. It follows that $\phi-\operatorname{Rk}(\Psi(\bar{x}) \cup\{\theta(\bar{x})\})=\phi-\operatorname{Rk}(\Psi(\bar{x}))$, as required.

Applying Lemma 7.1 in the context of complete $\exists \forall$-theories we obtain

7.3. Lemma. Let $T$ be a complete $\exists \forall$-theory over a relational language and $\mathscr{M} \vDash T$ be $\exists \forall$-saturated. Let $B \subseteq M, \phi(\bar{x}, \bar{y})$ be a $\exists: \forall$-formula with $l(\bar{x})=$ $m$, and $\Psi(\bar{x})$ be a set of $\exists: \forall$-formulas over $B$. There exists a finite equivalence relation $E$ on $M^{m}$ defined by $a \exists: \forall$-formula over $B$ such that for any $E$-class C

(1) either

$$
\phi-\operatorname{Rk}(\Psi(\bar{x}) \cup\{\bar{x} \in C\})<\phi-\operatorname{Rk}(\Psi(\bar{x}))
$$

or

$$
\phi-\operatorname{Mt}(\Psi(\bar{x}) \cup\{\bar{x} \in C\})=1 ;
$$

(2) there is a formula $\sigma(\bar{x})$ over $M$ based on $\phi(\bar{x}, \bar{y})$ such that $\Lambda \Psi(\bar{x})$ implies $\bar{x} \in C \leftrightarrow \sigma(\bar{x})$.

Proof. (1) Let $\mathscr{N} \supseteq_{\forall} \mathscr{M}$ be $\exists \forall$-saturated over $B$, i.e., for every finite $X \subseteq N$, every $\exists \forall$-type over $B \cup X$ is realized in $\mathscr{N}$. By Corollary $3.9, \mathscr{M} \preceq \mathscr{N}$. Let 
$\phi-\operatorname{Rk}(\Psi(\bar{x}))=r$. Since $\exists: \forall$-formulas are stable, there is a formula $\theta(\bar{x}, \bar{c})$ over $N$ based on $\phi(\bar{x}, \bar{y})$ such that $\phi-(\mathrm{Rk}, \operatorname{Mt})(\Psi(\bar{x}) \cup\{\theta(\bar{x}, \bar{c})\})=(r, 1)$. Let $\chi(\bar{x}, \bar{d})$ satisfy the conclusion of Lemma 7.1 with $\mathscr{N}$ for $\mathscr{M}$. Since $\phi-\operatorname{Rk}(\Psi(\bar{x}) \& \chi(\bar{x}, \bar{d}))=\phi-\operatorname{Rk}(\Psi(\bar{x}))$, there exist a least number $k<\omega$ and conjugates $\chi\left(\bar{x}, \bar{d}_{i}\right) \quad(i<k)$ of $\chi(\bar{x})$ over $B$ such that, in an elementary extension of $\mathscr{M}$, for every conjugate $\chi^{\prime}(\bar{x})$ of $\chi(\bar{x}, \bar{d})$ over $B$ there exists $i<$ $k$ such that $\bigwedge \Psi(\bar{x})$ implies $\chi^{\prime}(\bar{x}) \leftrightarrow \chi\left(\bar{x}, \bar{d}_{i}\right)$. Let $\Gamma(\bar{u})$ denote $\exists-\operatorname{tp}(\bar{d} \mid B) \cup$ $\forall-\operatorname{tp}(\bar{d} \mid B)$. From Corollary $3.9 \Gamma(\bar{u})$ implies $\operatorname{Tp}(\bar{d} \mid B)$ in $\mathscr{N}$. Thus the set of formulas

$$
\begin{gathered}
\Delta=\Gamma\left(\bar{u}_{0}\right) \cup \cdots \cup \Gamma\left(\bar{u}_{k}\right) \cup \bigcup\left\{\Psi\left(\bar{x}_{i j}\right): i<j \leq k\right\} \\
\cup\left\{\chi\left(\bar{x}_{i j}, \bar{u}_{i}\right) \leftrightarrow \neg \chi\left(\bar{x}_{i j}, \bar{u}_{j}\right): i<j \leq k\right\}
\end{gathered}
$$

is not satisfiable in $\mathscr{N}$. Let $D_{\forall}(\mathscr{N})$ denote the $\forall$-diagram of $\mathscr{N}$. Since $\mathscr{N}$ is $\exists \forall$-saturated over $B, \Delta \cup D_{\forall}(\mathscr{N})$ is not satisfiable. By compactness there exist $\gamma(\bar{u})$, a finite conjunction of formulas from $\Gamma(\bar{u})$, and $\psi(\bar{x})$, a finite conjunction of formulas from $\Psi(\bar{x})$, such that the same is still true if in $\Delta$ we replace $\Gamma\left(\bar{u}_{i}\right)$ by $\left\{\gamma\left(\bar{u}_{i}\right)\right\}$ and $\Psi\left(\bar{x}_{i j}\right)$ by $\left\{\psi\left(\bar{x}_{i j}\right)\right\}$.

The formulas

$$
\begin{aligned}
& \left(\neg \psi\left(\bar{x}_{1}\right) \& \neg \psi\left(\bar{x}_{2}\right)\right) \\
& \quad \vee\left(\psi\left(\bar{x}_{1}\right) \& \psi\left(\bar{x}_{2}\right) \& \exists \bar{u}_{1} \cdots \exists \bar{u}_{k} \exists \bar{x}_{12} \exists \bar{x}_{13} \ldots \ldots\right. \\
& \quad \exists \bar{x}_{k-1 k}\left[\bigwedge\left\{\gamma\left(\bar{u}_{i}\right): 1 \leq i \leq k\right\}\right. \\
& \left.\quad \& \bigwedge\left\{\psi\left(\bar{x}_{i j}\right) \&\left(\chi\left(\bar{x}_{i j}, \bar{u}_{i}\right)\right) \leftrightarrow \neg \chi\left(\bar{x}_{i j}, \bar{u}_{j}\right)\right): 1 \leq i<j \leq k\right\} \\
& \left.\left.\quad \& \bigwedge\left\{\chi\left(\bar{x}_{1}, \bar{u}_{i}\right) \leftrightarrow \chi\left(\bar{x}_{2}, \bar{u}_{j}\right): 1 \leq i \leq k\right\}\right]\right)
\end{aligned}
$$

and

$$
\left(\neg \psi\left(\bar{x}_{1}\right) \& \neg \psi\left(\bar{x}_{2}\right)\right) \vee\left(\psi\left(\bar{x}_{1}\right) \& \psi\left(\bar{x}_{2}\right) \& \forall \bar{u}\left[\gamma(\bar{u}) \rightarrow \chi\left(\bar{x}_{1}, \bar{u}\right) \leftrightarrow \chi\left(\bar{x}_{2}, \bar{u}\right)\right]\right)
$$

define the same finite equivalence relation $E$ on $N^{m}$. Since one of these formulas is equivalent to a $\exists \forall$-formula and the other to a $\forall \exists$-formula, $E$ is defined by some $\exists: \forall$-formula over $B$ by Corollary 3.9. Similarly, $\tau(\bar{x})=$ $\exists \bar{u}(\gamma(\bar{u}) \& \chi(\bar{x}, \bar{u}))$ is equivalent to a $\exists: \forall$-formula over $B$. Now $E$ satisfies the conclusion of the lemma for $\Psi(\bar{x} \cup\{\tau(\bar{x})\})$, because we started with the formula $\chi(\bar{x}, \bar{d})$ such that $\phi-(\mathrm{Rk}, \mathrm{Mt})(\Psi(\bar{x}) \cup\{\chi(\bar{x}, \bar{d})\})=(r, 1)$. Also either $\phi-\operatorname{Rk}(\Psi(\bar{x}) \cup\{\neg \tau(\bar{x})\})<\phi-\operatorname{Rk}(\Psi(\bar{x}))$ or $\phi-\operatorname{Mt}(\Psi(\bar{x}) \cup\{\neg \tau(\bar{x})\})<\phi-\operatorname{Mt}(\Psi(\bar{x}))$. In the former case $E$ will do for $\Psi(\bar{x})$, and in latter we can repeat the whole argument beginning with $\Psi(\bar{x}) \cup\{\neg \tau(\bar{x})\}$ instead of $\Psi(\bar{x})$. In a finite number of steps we obtain a new equivalence relation $E$ which works for $\Psi(\bar{x})$. Thus (1) is proved except that we worked in $\mathscr{N}$ instead of $\mathscr{M}$ which does not matter since $\mathscr{M} \preceq \mathscr{N}$.

(2) Without loss of generality suppose that the search for $E$ ends in one step. If $\bar{a}_{1}, \bar{a}_{2}$ are solutions of $\Psi(\bar{x})$, then $\bar{a}_{1} E \bar{a}_{2}$ if and only if $\vDash \chi\left(\bar{a}_{1}, \bar{d}_{1}\right) \leftrightarrow$ 
$\chi\left(\bar{a}_{2}, \bar{d}_{i}\right) \quad(i<k)$. Since $\chi(\bar{x}, \bar{d})$ is based on $\phi(\bar{x}, \bar{y}), \bar{x} \in C$ restricted to $\Psi(\bar{x})$ is equivalent to a formula based on $\phi(\bar{x}, \bar{y})$.

Consider a model $\mathscr{M}$ of a complete $\exists \forall$-theory $T$. For $\bar{a} \in M$ and $B \subseteq M$, $\exists: \forall-\operatorname{tp}(\bar{a} \mid B)$ denotes $\exists-\operatorname{tp}(\bar{a} \mid B) \cup \forall-\operatorname{tp}(\bar{a} \mid B)$. More generally, a $\exists: \forall$-type over $B$ is one of the form $\exists: \forall-\operatorname{tp}(\bar{a} \mid B)$, where $\bar{a} \in N$ and $\mathcal{N} \supseteq_{\forall} M$. Unless otherwise specified, $\exists: \forall$-types are complete. Here these types play the role of complete elementary types in ordinary stability theory.

Let $F E_{\exists: \forall}^{m}(B)$ denote the family of all finite equivalence relations on $M^{m}$ definable by $\exists: \forall$-formulas over $B$. Let $\bar{a} \in M$ and $l(\bar{a})=m$. We define

$$
\exists: \forall-\operatorname{stp}(\bar{a} \mid B)=\left\{E(\bar{x}, \bar{a}): E \in F E_{\exists: \forall}^{m}(B)\right\} .
$$

This is the $\exists: \forall$-strong type of $\bar{a}$ over $B$. In general, a $\exists: \forall$-strong type over $B$ is one of the form $\exists: \forall-\operatorname{stp}(\bar{a} \mid B)$ for $\bar{a}$ in some $\forall$-extension of $\mathscr{M}$. It is clear that every $\exists: \forall$-strong type over $B$ implies a unique $\exists \forall$-type over $B$.

7.4. Lemma. Let $\mathscr{M}$ be a $\exists \forall$-saturated model of a complete $\exists \forall$-theory $T$. Let $l(\bar{x})=m, B \subseteq M, \Psi(\bar{x})$ be a $\exists: \forall$-strong type over $B$, and $\Theta(\bar{\chi})$ be the corresponding $\exists: \forall$-type over $B$. Let $\phi(\chi, \bar{y})$ be a $\exists: \forall$-formula without parameters.

(1) $\phi-(\mathbf{R k}, \mathbf{M t})(\Psi(\bar{\chi}))=(\phi-\mathbf{R k}(\Theta(\bar{\chi})), 1)$.

(2) Let $\phi_{i}\left(\bar{\chi}, \bar{y}_{i}\right) \quad(i<k)$ be $\exists: \forall$-formulas without parameters and $\theta_{i}(i<$ $k)$ be a formula over $M$ based on $\phi_{i}\left(\bar{\chi}, \bar{y}_{i}\right)$ such that

$$
\phi_{i}-\operatorname{Rk}\left(\Psi(\bar{\chi}) \cup\left\{\theta_{i}(\bar{\chi})\right\}\right)<\phi_{i}-\operatorname{Rk}(\Psi(\bar{x})) \quad(i<k) .
$$

Then $\Psi(\bar{\chi}) \cup\left\{\neg \theta_{i}(\bar{\chi}): i<k\right\}$ has the same $\phi$-rank as $\Psi(\bar{\chi})$.

Proof. (1) Towards a contradiction suppose that $\phi-\operatorname{Rk}(\Psi(\bar{x}))<\phi-\operatorname{Rk}(\Theta(\bar{x}))$. Let $\bar{a}$ realize $\Psi(\bar{x})$, where $\bar{a}$ is in some $\forall$-extension of $\mathscr{M}$. Since $\phi$-rank has finite character there exist $n<\omega$ and $E_{i} \in F E_{\exists: \forall}^{m}(B)$ such that

$$
\phi-\operatorname{Rk}\left(\Theta(\bar{x}) \cup\left\{E_{i}(\bar{x}, \bar{a}): i<n\right\}\right)<\phi-\operatorname{Rk}(\Theta(\bar{x})) .
$$

There exists $E \in F E_{\exists: \forall}^{m}(B)$ such that

$$
\mathscr{M} \vDash \forall \bar{x}_{1} \forall \bar{x}_{2}\left[E\left(\bar{x}_{1}, \bar{x}_{2}\right) \rightarrow \bigwedge\left\{E_{i}\left(\bar{x}_{1}, \bar{x}_{2}\right): i<n\right\}\right] .
$$

Hence, $\phi-\operatorname{Rk}(\Theta(\bar{x}) \cup\{E(\bar{x}, \bar{a})\})<\phi-\operatorname{Rk}(\Theta(\bar{x}))$. Since $E$ is a finite equivalence relation there exist $k<\omega$ and $\bar{a}_{i} \in M(i<k)$ realizing $\Theta(\bar{x})$ such that $\bar{a}_{0}=\bar{a}$ and

$$
\mathscr{M} \vDash(\bigwedge \Theta(\bar{x})) \rightarrow \bigvee\left\{E\left(\bar{x}, \bar{a}_{i}: i<k\right)\right\}
$$

A basic property of $\phi$-rank tells us that for some $i<k$

$$
\phi-\operatorname{Rk}\left(\boldsymbol{\Theta}(\bar{x}) \cup\left\{E\left(\bar{x}, \bar{a}_{i}\right)\right\}\right)=\phi-\operatorname{Rk}(\Theta(\bar{x})) .
$$

Since each $\bar{a}_{i}$ realizes the same elementary type over $B$ in $\mathscr{M}$,

$$
\phi-\operatorname{Rk}\left(\Theta(\bar{x}) \cup\left\{E\left(\bar{x}, \bar{a}_{i}\right)\right\}\right)
$$


is the same for all $i<k$. We now have a contradiction. Thus, $\phi-\operatorname{Rk}(\Psi(\bar{x}))=$ $\phi-\operatorname{Rk}(\Theta(\bar{x}))$.

It remains to show that $\phi-\operatorname{Mt}(\Psi(\bar{x}))=1$. From Lemma 7.3 there exists $E \in F E_{\exists: \forall}^{m}(B)$ such that for each $m$-tuple $\bar{a} \in M$ either

$$
\phi-\operatorname{Rk}(\Theta(\bar{x}) \cup\{E(\bar{x}, \bar{a})\})<\phi-\operatorname{Rk}(\Theta(\bar{x}))
$$

or

$$
\phi-\operatorname{Mt}(\Theta(\bar{x}) \cup\{E(\bar{x}, \bar{a})\})=1 .
$$

Let $\bar{a}_{i} \in \Theta(\mathscr{M}) \quad(i<n)$ be representatives of all the $E$-classes which meet $\Theta(\mathscr{M})$. Since $\operatorname{Tp}\left(\bar{a}_{i} \mid B\right)$ is determined by $\Theta\left(\bar{a}_{i}\right), \phi-(\mathrm{Rk}, \mathrm{Mt})\left(\Theta(\bar{x}) \cup\left\{E\left(\bar{x}, \bar{a}_{i}\right)\right\}\right)$ does not depend on $i<k$. Since $\Theta(\bar{x})$ is covered by the formulas $E\left(\bar{x}, \bar{a}_{i}\right)$ $(i<k)$,

$$
\phi-\operatorname{Rk}\left(\Theta(\bar{x}) \cup\left\{E\left(\bar{x}, \bar{a}_{i}\right)\right\}\right)=\phi-\operatorname{Rk}(\Theta(\bar{x})) \quad(i<k) .
$$

It follows that $\phi-\operatorname{Mt}\left(\Theta(\bar{x}) \cup\left\{E\left(\bar{x}, \bar{a}_{i}\right)\right\}\right)=1 \quad(i<k)$. Since $\Lambda \Psi(\bar{x})$ implies $\Theta(\bar{x}) \cup\left\{E\left(\bar{x}, \bar{a}_{i}\right)\right\}$ for some $i<k$ and has the same $\phi$-rank, $\phi$ - $\operatorname{Mt}(\Psi(\bar{x}))=1$.

(2) Choose a $\exists: \forall$-formula $\phi^{\prime}\left(\bar{x}, \bar{y}^{\prime}\right)$ without parameters such that

$$
\vdash_{T} \forall \bar{y} \exists \bar{y}^{\prime} \forall \bar{x}\left[\phi(\bar{x}, \bar{y}) \leftrightarrow \phi^{\prime}\left(\bar{x}, \bar{y}^{\prime}\right)\right]
$$

and for all $i<k$

$$
\vdash_{T} \forall \bar{y}_{i} \exists \bar{y}^{\prime} \forall \bar{x}\left[\phi_{i}\left(\bar{x}, \bar{y}_{i}\right) \leftrightarrow \phi^{\prime}\left(\bar{x}, \bar{y}^{\prime}\right)\right] .
$$

Let $\Theta^{\prime}(\bar{x})$ be the complete elementary type determined by $\Theta(\bar{x})$ in $\exists \forall$-saturated $\forall$-extensions of $\mathscr{M}$. From Lemma 7.3 there is a formula $\sigma_{i}(\bar{x})$ over $M$ based on $\phi_{i}\left(\bar{x}, \bar{y}_{i}\right)$ such that $\Psi(\bar{x})$ contains a formula equivalent to $\sigma_{i}(\bar{x})$, $\phi_{i}-\operatorname{Rk}\left(\Theta(\bar{x}) \cup\left\{\sigma_{i}(\bar{x})\right\}\right)=\phi_{i}-\operatorname{Rk}(\Theta(\bar{x}))$, and $\phi_{i}-\operatorname{Mt}\left(\Theta(\bar{x}) \cup\left\{\sigma_{i}(\bar{x})\right\}\right)=1$. Notice that essentially $\Theta(\bar{x}) \cup\left\{\sigma_{i}(\bar{x})\right\} \subseteq \Psi(\bar{x})$. From (1), $\phi_{i}-(\mathbf{R k}, \mathbf{M t})(\Psi(\bar{x}))=$ $\left(\phi_{i}-\mathbf{R} \mathbf{k}(\Theta(\bar{x})), 1\right)$. Since $\phi_{i}-\operatorname{Rk}\left(\Psi(\bar{x}) \cup\left\{\theta_{i}(\bar{x})\right\}\right)<\phi_{i}-\operatorname{Rk}(\Psi(\bar{x}))$, we have

$$
\phi_{i}-\operatorname{Rk}\left(\Theta(\bar{x}) \cup\left\{\theta_{i}(\bar{x}), \sigma_{i}(\bar{x})\right\}\right)<\phi_{i}-\operatorname{Rk}(\Theta(\bar{x})) .
$$

Since $\Lambda \Theta(\bar{x})$ and $\wedge \Theta^{\prime}(\bar{x})$ have the same solution set in every $\exists \forall$-saturated $\forall$-extension of $\mathscr{M}$, we can substitute $\Theta^{\prime}(\bar{x})$ for $\Theta(\bar{x})$ in $(*)$. Applying Lemma 7.2 yields

$$
\phi^{\prime}-\operatorname{Rk}\left(\Theta^{\prime}(\bar{x}) \cup\left\{\theta_{i}(\bar{x}), \sigma_{i}(\bar{x})\right\}\right)<\phi^{\prime}-\mathbf{R k}\left(\Theta^{\prime}(\bar{x})\right) .
$$

and reversing the replacements gives

$$
\begin{aligned}
\phi^{\prime}-\mathbf{R k}\left(\Psi(\bar{x}) \cup\left\{\theta_{i}(\bar{x})\right\}\right) & \leq \phi^{\prime}-\mathbf{R k}\left(\Theta(\bar{x}) \cup\left\{\theta_{i}(\bar{x}), \sigma_{i}(\bar{x})\right\}\right) \\
& <\phi^{\prime}-\operatorname{Rk}(\Theta(\bar{x}))=\phi^{\prime}-\mathbf{R k}(\Psi(\bar{x})) .
\end{aligned}
$$

It follows immediately that $\Psi(\bar{x}) \cup\left\{\neg \theta_{i}(\bar{x}): i<k\right\}$ has the same $\phi^{\prime}$-rank as $\Psi(\bar{x})$. Towards a contradiction suppose that

$$
\phi-\operatorname{Rk}\left(\Psi(\bar{x}) \cup\left\{\neg \theta_{i}(\bar{x}): i<k\right\}\right)<\phi-\operatorname{Rk}(\Psi(\bar{x})) .
$$

Then

$$
\phi-\operatorname{Rk}\left(\Psi(\bar{x}) \cup\left\{\bigwedge\left\{\neg \theta_{i}(\bar{x}): i<k\right\}\right\}\right)<\phi-\operatorname{Rk}(\Psi(\bar{x})) .
$$


Repeating the argument made above we see that $\Psi(\bar{x}) \cup\left\{\neg \wedge\left\{\neg \theta_{i}(\bar{x}): i<k\right\}\right\}$ has the same $\phi^{\prime}$-rank as $\Psi(\bar{x})$, whence $\Psi(\bar{x}) \cup\left\{\theta_{i}(\bar{x})\right\}$ has the same $\phi^{\prime}$-rank as $\Psi(\bar{x})$ for some $i<k$. This contradicts (\#) above and completes the proof of the lemma.

We now have all the underpinnings for what one might call $\exists: \forall$-stability theory. Let $T$ be a complete $\exists \forall$-theory, $\mathscr{M} \vDash T, B \subseteq C \subseteq M, l(\bar{x})=m$, and $\Phi(\bar{x})$ be a $\exists: \forall$-strong type over $B$. The heir $\Theta(\bar{x})$ over $C$ of $\Phi(\bar{x})$ is defined as follows. Let $\mathscr{N} \supseteq_{\forall} \mathscr{M}$ be $\exists \forall$-saturated and $\Theta(\bar{x})=\left\{E(\bar{x}, \bar{a}): E \in E F_{\exists: \forall}^{m}(C), \bar{a} \in N, \phi(\bar{x}, \bar{y})\right.$ is a $\exists: \forall$-formula, $E(\bar{x}, \bar{a})$ is based on $\phi(\bar{x}, \bar{y}), \phi-\operatorname{Rk}(\Phi(\bar{x}) \cup\{E(\bar{x}, \bar{a})\})=\phi-\operatorname{Rk}(\Phi(\bar{x}))\}$.

From Lemma 7.4 we see that $\Theta(\bar{x}) \supseteq \Phi(\bar{x})$ is a $\exists: \forall$-strong type over $C$ and that $\phi-\operatorname{Rk}(\Theta(\bar{x}))=\phi-\operatorname{Rk}(\Phi(\bar{x}))$ for every $\exists: \forall$-formula $\phi(\bar{x}, \bar{y})$. Moreover, $\Theta(\bar{x})$ is the unique such $\exists: \forall$-strong type.

Let $\mathscr{M} \vDash T$ and $A, B, C \subseteq M$. Then $A$ and $C$ are independent over $B$, written $A \downarrow C(B)$, if in some $\exists \forall$-saturated model $\mathscr{N} \supseteq_{\forall} \mathscr{M}, \exists: \forall$ $\operatorname{stp}(\bar{a} \mid B \cup C)$ is the heir of $\exists: \forall-\operatorname{stp}(\bar{a} \mid B)$ for all $\bar{a} \in A$. By Corollary 3.9 writing "every" for "some" makes no difference. This relation has the familiar properties:

Finite character $A \downarrow C(B)$ if and only if for all finite $A^{\prime} \subseteq A$ and all finite $C^{\prime} \subseteq C$ we have $A^{\prime} \downarrow C^{\prime}(B)$. Moreover, if $\bar{a}$ and $\bar{c}$ are dependent over $B$, meaning that $\bar{a} \downarrow \bar{c}(B)$ fails, then there is a formula $\theta(\bar{x}, \bar{z})$ over $B$ such that $\vDash \theta(\bar{a}, \bar{c})$ and

$$
[\theta(\bar{x}, \bar{z}) \&(\bar{x}, \bar{z} \text { realize } \exists: \forall-\operatorname{stp}(\bar{a} \mid B), \exists: \forall-\operatorname{stp}(\bar{c} \mid B))] \rightarrow \neg[\bar{x} \downarrow \bar{z}(B)] .
$$

(We could replace $\exists: \forall$-stp by $\exists: \forall$-tp here which would sharpen the result.)

Transitivity $A \downarrow C \cup D(B)$ if and only if $A \downarrow B \cup C \quad(B)$ and $A \downarrow D$ $(B \cup C)$.

Symmetry $A \downarrow C \quad(B)$ if and only if $C \downarrow A \quad(B)$.

Trivial independence $A \downarrow C(A)$.

Let $B \subseteq M, l(\bar{x})=m$, and $\Phi(\bar{x})$ be a $\exists: \forall$-strong type over $B$. A sequence $\left\langle\bar{a}_{i}: i<\alpha\right\rangle$, where $\bar{a}_{i} \in N^{m}$ and $\mathscr{N} \supseteq_{\forall} \mathscr{M}$ is a Morley sequence for $\Phi(\bar{x})$ if $\exists: \forall-\operatorname{stp}\left(\bar{a}_{j} \mid B \cup \bigcup\left\{\bar{a}_{i}: i<j\right\}\right)$ is the heir of $\Phi(\bar{x})$ for all $j<\alpha$. Such a Morley sequence is said to be over $B$. The $\exists: \forall$-type over $B$ of a Morley sequence over $B$ is uniquely determined by $\Phi(\bar{x})$, and the set of $m$-tuples enumerated by such a sequence is indiscernible with respect to $\exists: \forall$-formulas over $B$. Given $\bar{a} \in M$ and $\alpha \in \mathrm{On}$, in a suitable $\forall$-extension $\mathscr{N}$ of $\mathscr{M}$ there exists a Morley sequence $\left\langle\bar{a}_{i}: i<\alpha\right\rangle$ for $\exists: \forall-\operatorname{stp}(\bar{a} \mid B)$ with $\bar{a}_{0}=\bar{a}$.

The following lemma relates Morley sequences to dependence and dividing. Since the situation is exactly parallel to that in ordinary stability theory, the proof is left to the reader.

7.5. Lemma. Let $\mathscr{M}$ be a model of a complete $\exists \forall$-theory $T$. Let $B \subseteq M$, and $\Phi(\bar{x}), \Theta(\bar{z})$ be $\exists: \forall$-strong types over $B$ realized by $\bar{a}, \bar{c}$ respectively. Let $\bar{a}_{0}, \bar{a}_{1}, \ldots$ be a Morley sequence of $\Phi(\bar{x})$. 
(1) $\bar{a}$ and $\bar{c}$ are dependent over $B$ if and only if there exist $\exists: \forall$-formulas $\phi(\bar{z}, \bar{y})$ without parameters and $\psi(\bar{x}, \bar{z})$ over $B$ such that $\mathscr{M} \vDash \psi(\bar{a}, \bar{c})$ and $\phi-\mathbf{R k}(\boldsymbol{\Theta}(\bar{z}) \cup\{\psi(\bar{a}, \bar{z})\})<\phi-\operatorname{Rk}(\boldsymbol{\Theta}(\bar{z}))$.

(2) If $\phi(\bar{z}, \bar{y})$ without parameters and $\psi(\bar{x}, \bar{z}))$ over $B$ are $\exists: \forall$-formulas such that $\phi-\operatorname{Rk}(\Theta(\bar{z}) \cup\{\psi(\bar{a}, \bar{z})\})<\phi-\operatorname{Rk}(\Theta(\bar{z}))$, then the formulas $\psi\left(\bar{a}_{i}, \bar{z}\right)$ $(i<\omega)$ are almost disjoint in $\Theta(\bar{z})$.

(3) If $\phi(\bar{z}, \bar{y})$ without parameters and $\psi(\bar{x}, \bar{z})$ over $B$ are $\exists: \forall$-formulas such that the formulas $\psi\left(\bar{a}_{i}, \bar{z}\right) \quad(i<\omega)$ are almost disjoint in $\Theta(\bar{z})$, and $\psi(\bar{a}, \bar{z})$ is based on $\phi(\bar{z}, \bar{y})$, then $\phi-\operatorname{Rk}(\Theta(\bar{z}) \cup\{\psi(\bar{a}, \bar{z})\})<\phi-\operatorname{Rk}(\Theta(\bar{z}))$.

We now come to the crucial result which will enable us to show that the theories we are studying are tree-decomposable.

7.6. Lemma (Triviality of independence). For all $\mathscr{M} \vDash T, A, C, D \subseteq M$, and $b \in M$

$$
[A \downarrow C(D)] \Rightarrow[A \downarrow b(C \cup D) \text { or } C \downarrow b(A \cup D)] \text {. }
$$

Proof. Towards a contradiction let $A, C, D, b$ constitute a counterexample. From the finite character of independence we can assume that there are finite tuples $\bar{a}, \bar{c}$ such that $A=\bar{a}$ and $C=\bar{c}$. Let $c$ realize $\exists: \forall-\operatorname{stp}(b \mid C \cup D)$ and $c \downarrow A \cup\{b\} \quad(C \cup D)$. Clearly, $C \cup\{c\} \downarrow b \quad(A \cup D)$ fails since $C \downarrow b \quad(A \cup D)$ fails. By applying the listed properties of independence, principally transitivity, we deduce $C \cup\{c\} \downarrow A(D)$ and

$$
[A \downarrow b(C \cup\{c\} \cup D)] \Rightarrow[A \downarrow b(C \cup D)] .
$$

Thus without spoiling the counterexample we can adjoin $c$ to $C$. So we can suppose that $\Psi(y)=\exists: \forall-\operatorname{tp}(b \mid C \cup D)$ is equivalent to the $\exists: \forall$-strong type of $b$ over $C \cup D$, and similarly with $A$ instead of $C$.

Let $\Phi(\bar{x})$ and $\Theta(\bar{z})$ be the $\exists: \forall$-strong types over $D$ realized by $\bar{a}$ and $\bar{c}$. Let $\bar{a}_{0}(=\bar{a}), \bar{a}_{1}, \ldots$ and $\bar{c}_{0}(=\bar{c}), \bar{c}_{1}, \ldots$ be Morley sequences for $\Phi(\bar{x})$ and $\Theta(\bar{z})$ such that

$$
\bigcup\left\{\bar{a}_{1}: i<\omega\right\} \downarrow \bigcup\left\{\bar{b}_{i}: i<\omega\right\}(D) .
$$

Since $\neg[A \downarrow b \quad(C \cup D)]$, from Lemma 7.5 there is a $\exists: \forall$-formula $\sigma(y, \bar{x}, \bar{z})$ over $D$ such that $\mathscr{M} \vDash \sigma(b, \bar{a}, \bar{c})$ and the formulas $\sigma\left(y, \bar{a}_{i}, \bar{c}\right) \quad(i<\omega)$ are almost disjoint in $\Psi(\mathscr{M})$. By compactness there is a formula $\psi(y) \in \Psi(y)$ such that the formulas $\sigma\left(y, \bar{a}_{i}, \bar{c}\right) \quad(i<\omega)$ are almost disjoint in $\psi(\mathscr{M})$. Thus, $\sigma(y, \bar{x}, \bar{z})$ can be chosen so that the formulas $\sigma\left(y, \bar{a}_{i}, \bar{c}\right)$ are almost disjoint (in $\mathscr{M})$. Similarly we see that there is a $\exists: \forall$-formula $\pi(y, \bar{x}, \bar{z})$ over $D$ such that $\mathscr{M} \vDash \pi(b, \bar{a}, \bar{c})$ and the formulas $\pi\left(y, \bar{a}, \bar{c}_{i}\right) \quad(i<\omega)$ are almost disjoint. Taking the conjunction of $\sigma$ and $\pi$ we obtain a $\exists: \forall$-formula $\chi(y, \bar{x}, \bar{z})$ over $D$ such that $\mathscr{M} \vDash \exists y \chi(y, \bar{a}, \bar{c})$, and both the formulas $\chi\left(y, \bar{a}_{i}, \bar{c}\right) \quad(i<\omega)$ and $\chi\left(y, \bar{a}, \bar{c}_{i}\right) \quad(i<\omega)$ are almost disjoint.

Below we shall show that the configuration afforded by $D$, the $\exists: \forall$-strong types $\Phi(\bar{x})$ and $\Theta(\bar{z})$, and the formula $\chi(y, \bar{x}, \bar{z})$, is not compatible with $T$ 
being a complete $\exists \forall$-theory. However, before we proceed it is convenient to replace the language by a finite sublanguage (the relation symbols occurring in $\chi$ will suffice), $T$ by its restriction to the new language, $D$ by the finite subset of elements whose names appear in $\chi$, and $\mathscr{M}$ by a countable elementary substructure of the $\exists \forall$-saturated model considered above. Below, the finite language chosen will be referred to as $L$. Thus, assuming that the lemma fails, we have:

7.6.1. Proposition. There exists a countable $\exists \forall$-closed model $\mathscr{M}, D \subseteq \subseteq_{\text {fin }} M$, $\exists: \forall$-strong types $\Phi(\bar{\chi})$ and $\Theta(\bar{z})$ over $D$, and $a \exists: \forall$-formula $\chi(y, \bar{\chi}, \bar{z})$ over $D$ such that, if $\left\langle\bar{a}_{i}: i<\omega\right\rangle$ and $\left\langle\bar{c}_{i}: i<\omega\right\rangle$ are mutually independent Morely sequences for $\Phi(\bar{\chi})$ and $\Theta(\bar{z})$ in a $\exists \forall$-closed $\forall$-extension $\mathscr{M}^{\prime}$ of $\mathscr{M}$, then $\bar{a}_{i} \cap \bar{a}_{j}=\bar{c}_{i} \cap \bar{c}_{j}=\varnothing \quad(i<j<\omega), \mathscr{M}^{\prime} \vDash \exists y \chi\left(y, \bar{a}_{i}, \bar{c}_{j}\right) \quad(i, j<\omega)$, the formulas $\chi\left(y, \bar{a}_{i}, \bar{c}_{j}\right) \quad(j<\omega)$ are almost disjoint in $\mathscr{M}^{\prime}$ for each $i$, and the formulas $\chi\left(y, \bar{a}_{i}, \bar{c}_{j}\right) \quad(i<\omega)$ are almost disjoint in $\mathscr{M}^{\prime}$ for each $j$.

Let $\mathscr{M}$, now countable, be elementarily embedded in a highly $\exists \forall$-saturated model $\mathscr{C}$. Let constants be introduced naming the elements of $\mathscr{M}$. Let $\mathbb{Q}$ denote the set of rationals, $\bar{a}_{q} \in C \backslash M(q \in \mathbb{Q})$ realize the heir of $\Phi(\bar{x})$ over $M \cup \cup\left\{\bar{a}_{r}: r \in \mathbb{Q} \backslash\{q\}\right\}$, and $\bar{c}_{q} \in C \backslash M \quad(q \in \mathbb{Q})$ realize the heir of $\Theta(\bar{z})$ over $M \cup \bigcup\left\{\bar{c}_{r}: r \in \mathbb{Q} \backslash\{q\}\right\} \cup \bigcup\left\{\bar{a}_{r}: r \in \mathbb{Q}\right\}$. Without loss of generality $\bar{a}_{q} \cap \bar{c}_{r}=\varnothing$ $(q, r \in \mathbb{Q})$. Note that the sets $\left\{\bar{a}_{r}: r \in \mathbb{Q}\right\}$ and $\left\{\bar{c}_{r}: r \in \mathbb{Q}\right\}$ are mutually independent over $M$, and hence mutually indiscernible over $M$ in $\mathscr{C}$.

For each formula of $L$, augmented with names for the elements of $M$, of the form

$$
\exists y_{1} \ldots \exists y_{m} \phi\left(y_{1}, \ldots, y_{m}, \bar{x}_{1}, \ldots, \bar{x}_{n}\right)
$$

with $\phi$ quantifier-free, $l\left(\bar{x}_{j}\right)=l(\bar{x}) \quad(1 \leq j \leq n)$, and

$$
\mathscr{C} \vDash \exists y_{1} \ldots \exists y_{m} \phi\left(y_{1}, \ldots, y_{m}, \bar{a}_{1}, \ldots, \bar{a}_{n}\right),
$$

we introduce Skolem functions $F_{1}^{\phi}, \ldots, F_{m}^{\phi}$ as follows. $F_{j}^{\phi}$ has arity $n \cdot l(\bar{x})$ and is defined only on tuples of the form $\bar{a}_{q_{1}} \cdots \bar{a}_{q_{n}}$ such that $q_{1}<\cdots<q_{n}$ in $\mathbb{Q}$. The values are such that

$$
\mathscr{C} \vDash \phi\left(F_{1}^{\phi}\left(\bar{a}_{q_{1}}, \ldots, \bar{a}_{q_{n}}\right), \ldots, F_{m}^{\phi}\left(\bar{a}_{q_{1}}, \ldots, \bar{a}_{q_{n}}\right), \bar{a}_{q_{1}}, \ldots, \bar{a}_{q_{n}}\right) .
$$

Similarly, for each formula over $M$ of the form

$$
\exists y_{1} \ldots \exists y_{m} \psi\left(y_{1}, \ldots, y_{m}, \bar{z}_{1}, \ldots, \bar{z}_{n}\right)
$$

with $\psi$ quantifier-free, $l\left(\bar{z}_{j}\right)=l(\bar{z}) \quad(1<j<n)$, and

$$
\mathscr{C} \vDash \exists y_{1} \ldots \exists y_{m} \psi\left(y_{1}, \ldots, y_{m}, \bar{c}_{1}, \ldots, \bar{c}_{n}\right),
$$

we introduce Skolem functions $G_{1}^{\psi}, \ldots, G_{m}^{\psi}$.

Let $L^{\prime}$ be $L$ augmented with names for the elements of $M \cup \cup\left\{\bar{a}_{r} \cup \bar{c}_{r}: r \in\right.$ $\mathbb{Q}\}$ and symbols $f_{j}^{\phi}, g_{j}^{\psi}$ for the Skolem functions just mentioned. The only terms allowed are variables, the names just mentioned, and those of the forms 
$f_{j}^{\phi} \bar{a}_{q_{1}} \cdots \bar{a}_{q_{n}}$ and $g_{j}^{\psi} \bar{c}_{q_{1}} \cdots \bar{c}_{q_{n}}$, where $q_{1}<\cdots<q_{n}$ in $\mathbb{Q}$. Let $\mathbf{A}=(F, G)$ be any pair of order automorphisms of $\mathbb{Q}$. We also denote by $\mathbf{A}$ the permutation of the variable-free terms of $L^{\prime}$ which fixes $M$ pointwise, maps $\bar{a}_{q}$ to $\bar{a}_{F(q)}$, $\bar{c}_{q}$ to $\bar{c}_{G(q)}$, and the other terms analogously. Enlarging $\mathscr{C}$ if necessary, we can choose $\bar{a}_{q}, \bar{c}_{q}$ and the values of the Skolem functions such that, for all order automorphisms $F, G$ of $\mathbb{Q}, \mathbf{A}=(F, G)$ preserves the truth-value of atomic sentences of $L^{\prime}$.

Let $l$ be an irrational, $0<l<1$, and $\mathscr{N}$ be the submodel of $\mathscr{C}$ such that $N \subseteq C$ is the least set satisfying:

$$
M \cup \bar{a}_{q} \cup \bar{c}_{q} \subseteq N \quad(-l<q \in \mathbb{Q})
$$

and

$$
F_{j}^{\phi}\left(\bar{a}_{q_{1}}, \ldots, \bar{a}_{q_{n}}\right), G_{j}^{\psi}\left(\bar{c}_{q_{1}}, \ldots, \bar{c}_{q_{n}}\right) \in N
$$

whenever there exists $k<\omega$ with $k-l<q_{1}<\cdots<q_{n}<k-l+1$. The elements of $N \backslash M$ are naturally partitioned into an $\omega$-sequence of $a$-blocks and an $\omega$-sequence of $c$-blocks. Let $\bar{a}^{k}$ and $\bar{c}^{k}$ enumerate the $k$ th $a$-block and $k$ th $c$-block respectively in such a way that $\bar{a}_{k}$ is an initial segement of $\bar{a}^{k}$, $\bar{c}_{k}$ is an initial segment of $\bar{c}^{k},\left\langle\bar{a}^{k}: k<\omega\right\rangle$ is order-indiscernible with respect to quantifier-free formulas over $M \cup \bigcup\left\{\bar{c}^{k}: k<\omega\right\}$, and $\left\langle\bar{c}^{k}: k<\omega\right\rangle$ is orderindiscernible with respect to quantifier-free formulas over $M \cup \bigcup\left\{\bar{a}^{k}: k<\omega\right\}$. Thus, assuming the lemma fails, we have:

7.6.2. Proposition. There exist $\mathscr{M}, D \subseteq M, \Phi(\bar{x}), \Theta(\bar{z})$, and $\chi(y, \bar{x}, \bar{z})$ satisfying the previous proposition, and $\mathscr{N} \supseteq_{\forall} \mathscr{M}$ such that:

(1) There exist tuples $\bar{a}^{i}, \bar{c}^{i} \quad(i<\omega)$ of length $\omega$ such that

$$
N \backslash M=\bigcup\left\{\bar{a}^{i} \cup \bar{c}^{i}: i<\omega\right\} .
$$

The set $\left\{\bar{a}^{i}: i<\omega\right\}$ is indiscernible in $\mathscr{N}$ over $M \cup \bigcup\left\{\bar{c}^{i}: i<\omega\right\}$, and $\left\{\bar{c}^{i}: i<\right.$ $\omega\}$ is indiscernible in $\mathscr{N}$ ovr $M \cup \bigcup\left\{\bar{a}^{i}: i<\omega\right\}$.

(2) Let $\bar{a}^{i}=\left(a_{0}^{i}, a_{1}^{i}, \ldots\right), \bar{c}^{i}=\left(c_{0}^{i}, c_{1}^{i}, \ldots\right)$, and $\bar{a}_{i}, \bar{c}_{i}$ denote $\left(a_{0}^{i}, \ldots\right.$, $\left.a_{l(\bar{x})-1}^{i}\right),\left(c_{0}^{i}, \ldots, c_{l(\bar{z})-1}^{i}\right)$ respectively. In $\mathcal{N},\left\langle\bar{a}_{i}: i<\omega\right\rangle$ is a Morley sequence for the heir of $\Phi(\bar{x})$ over $M$, and $\left\langle\bar{c}_{i}: i<\omega\right\rangle$ is a Morley sequence for the heir of $\Theta(\bar{z})$ over $M$.

Most of the points of the proposition are probably clear. However, one point is worth stressing. In $\mathscr{N}$ the tuples of the indexed set $A_{k}=\left\{\bar{a}_{q}: k-\imath<q<\right.$ $k-l+1\}$ satisfy exactly the same $\exists: \forall$-formulas over $M$ as in $\mathscr{C}$. This is the point of Skolem functions. Hence, each member of $A_{k}$ is independent from the rest of the set over $M$. Let $F$ be an order automorphism of $\mathbb{Q}$ such that $F(q)=q$ for all $q$ such that $q<k-l$ or $k-l+1<q$. The map $\bar{a}_{q} \mapsto \bar{a}_{F(q)}$ $(k-l<q<k-l+1)$ can be extended to an automorphism of $\mathscr{N}$ which fixes $M \cup \bigcup\left\{\bar{a}_{i}: i<\omega, i \neq k\right\}$ pointwise. Thus for every $q, k-l<q<k-l+1, \bar{a}_{q}$ realizes the heir of $\Phi(\bar{x})$ over $M \cup \bigcup\left\{\operatorname{Rng}\left(\bar{a}_{i}\right): i<\omega, i \neq k\right\}$. This explains 
why the $\bar{a}_{i}$ 's form a Morley sequence for the heir of $\Phi(\bar{x})$ over $M$. The same argument applies to the $\bar{c}_{i}$ 's. Another point to notice is that the orderindiscernibility of the $\bar{a}^{i}$ 's and $\bar{c}^{i}$ 's with respect to quantifier-free formulas yields indiscernibility because quantifier-free formulas are stable.

We are now ready for the main contradiction argument based on the models $\mathscr{M}$ and $\mathscr{N}$ of 7.6.2. Let $\bar{a}, \bar{c}$ denote $\bar{a}_{0}, \bar{c}_{0}$ respectively. Note that the Morley sequences $\left\langle\bar{a}_{i}: i<\omega\right\rangle$ and $\left\langle\bar{c}_{i}: i<\omega\right\rangle$ are mutually independent over $M$ because of the indiscernibility. From the symmetries of $\mathscr{N}$, any solution of $\chi(y, \bar{a}, \bar{c})$ in $\mathcal{N}$ is either a solution of all the formulas $\chi\left(y, \bar{a}, \bar{c}_{i}\right) \quad(i<\omega)$, or of all the formulas $\chi\left(y, \bar{a}_{i}, \bar{c}\right) \quad(i<\omega)$. Hence, $\chi(y, \bar{a}, \bar{c})$ has no solution in $\mathscr{N}$. However, by choice of $\chi$ there is a solution of $\chi(y, \bar{a}, \bar{c})$ in every $\exists \forall$-closed $\forall$-extension of $\mathcal{N}$.

Let $\chi(y, \bar{x}, \bar{z})$ be $\exists \bar{u} \phi(y, \bar{x}, \bar{z}, \bar{u}) \& \forall \bar{v} \psi(y, \bar{x}, \bar{z}, \bar{v})$, where $\phi$ and $\psi$ are quantifier-free formulas over $D$. Let $\left\langle D_{i}: i\langle\omega\rangle\right.$ be an increasing sequence of finite subsets of $N$ such that $\bar{a} \bar{c} \subseteq D_{0}, \bigcup_{i<\omega} D_{i}=N$, and for all $j, k \leq i$, $D_{i}$ is invariant under the permutation which switches $\bar{a}^{2 j}$ and $\bar{a}^{2 k}$ and fixes the rest of $N$ pointwise, and invariant under the permutation which switches $\bar{c}^{2 j}$ and $\bar{c}^{2 k}$ and fixes the rest of $N$ pointwise. Since $\chi(y, \bar{a}, \bar{c})$ has a solution in a $\forall$-extension of $\mathcal{N}$, for all $i<\omega$ there exist $b_{i}$ and $\bar{e}_{i}$ such that

$$
\mathscr{N} \vDash \phi\left(b_{i}, \bar{a}, \bar{c}, \bar{e}_{i}\right) \&\left(\forall \bar{v} \in D_{i} \cup b_{i} \bar{e}_{i}\right) \psi\left(b_{i}, \bar{a}, \bar{c}, \bar{v}\right) .
$$

Let $A, C$ denote $\bigcup\left\{\bar{a}^{i}: i<\omega\right\}, \bigcup\left\{\bar{c}^{i}: i<\omega\right\}$ respectively. (The model $\mathscr{C}$ has been forgotten.) Either $b_{i} \in A \cup M$ for infinitely many $i$, or $b_{i} \in C \cup M$ for infinitely many $i$. Without loss of generality, by thinning the sequence $\left\langle D_{i}: i<\right.$ $\omega\rangle$ we may suppose $b_{i} \in C \cup M$ for all $i$. Let $A^{\text {odd }}$ denote $\bigcup\left\{\bar{a}^{2 i+1}: i<\omega\right\}$.

We stipulate that $b_{i} \in C \cup M$ and $\bar{e}_{i}$ be chosen such that the number of entries of $\bar{e}_{i}$ which fall in $\bar{a}^{0}$ is as small as possible consistent with $(*)$. The number of entries of a tuple $\bar{e}$ which fall in $\bar{a}^{0}$ will be denoted by $n(\bar{e})$. By deleting an initial segment of $\left\langle D_{i}: i<\omega\right\rangle$ we ensure that $n\left(\bar{e}_{i}\right)$ is independent of $i$, equal to $s$ say.

Claim. $b_{i}$ and $\bar{e}_{i}$ may be chosen such that $b_{i} \in C \cup M,(\forall j>0)\left[\bar{a}^{j} \cap \bar{e}_{i}=\right.$ $\left.\varnothing \vee \bar{a}^{j} \cap D_{i}=\varnothing\right]$, and $\bar{e}_{i} \cap\left(A \backslash \bar{a}_{0}\right) \subseteq A^{\text {odd }}$.

Towards establishing the Claim fix $i$, choose $k$ such that $D_{i} \cap A \subseteq \bar{a}^{0} \cup \bar{a}^{1} \cup$ $\cdots \cup \bar{a}^{k}$, and let $D_{i}^{(n)}$ be obtained from $D_{i}$ by applying the map which takes $\bar{a}^{1}, \bar{a}^{2}, \ldots, \bar{a}^{k}$ to $\bar{a}^{n k+1}, \bar{a}^{n k+2}, \ldots, \bar{a}^{(n+1) k}$ respectively, and fixes $C \cup M \cup \bar{a}^{0}$ pointwise. Consider $j \in \omega$ such that

$$
\bigcup\left\{D_{i}^{(n)}: n \leq l(\bar{u})\right\} \subseteq D_{j}
$$

Clearly, for all $n \leq l(\bar{u})$,

$$
\mathcal{N} \vDash \phi\left(b_{j}, \bar{a}, \bar{c}, \bar{e}_{j}\right) \&\left(\forall \bar{v} \subseteq D_{i}^{(n)} \cup b_{j} \bar{e}_{j}\right) \psi\left(b_{j}, \bar{a}, \bar{c}, \bar{v}\right) .
$$


Since $\left|\bar{e}_{j}\right| \leq l(\bar{u})$ and

$$
(\forall h>0)(\forall m, n)\left[m<n \leq l(\bar{u}) \rightarrow\left(\bar{a}^{h} \cap D_{i}^{(m)}=\varnothing \vee \bar{a}^{h} \cap D_{i}^{(n)}=\varnothing\right)\right],
$$

there exists $m \leq l(\bar{u})$ such that $(\forall h>0)\left[\bar{a}^{h} \cap \bar{e}_{j}=\varnothing \vee \bar{a}^{h} \cap D_{i}^{(m)}=\varnothing\right]$. Since the set $\left\{\bar{a}^{0}, \bar{a}^{1}, \ldots\right\}$ is indiscernible over $C \cup M$, by switching $\bar{a}^{n}$ with $\bar{a}^{m k+n}$ for $1 \leq n \leq k$ and fixing all other elements of $N$, we can map $D_{i}^{(m)}$ onto $D_{i}$. Thus, we can choose $b_{i}$ and $\bar{e}_{i}$ such that $b_{i} \in C \cup M$ and $(\forall h>0)\left[\bar{a}^{h} \cap \bar{e}_{i}=\right.$ $\left.\varnothing \vee \bar{a}^{h} \cap D_{i}=\varnothing\right]$. By permuting the $\bar{a}^{h}$ such that $h>0$ and $\bar{a}^{h} \cap D_{i}=\varnothing$ we can ensure that $\bar{e}_{i} \cap\left(A \backslash \bar{a}^{0}\right) \subseteq A^{\text {odd }}$.

Let $b_{i}$ and $\bar{e}_{i}$ be chosen satisfying the conclusion of the Claim. Since $b_{0}$ and $\bar{e}_{0}$ have been chosen to minimize $n\left(\bar{e}^{0}\right)=s$, we have

$$
\mathscr{N} \vDash \forall \bar{u}\left[\left\{\phi\left(b_{i}, \bar{a}, \bar{c}, \bar{u}\right) \& n(\bar{u})<s\right\} \Rightarrow\left(\exists \bar{v} \subseteq D_{0} \cup \bar{u} b_{i}\right) \neg \psi\left(b_{i}, \bar{a}, \bar{c}, \bar{v}\right)\right] .
$$

Let $\bar{a}^{\prime}$ be a finite initial segment of $\bar{a}^{0}$ such that $\left(D_{0} \cap \bar{a}^{0}\right) \cup \bar{a} \subseteq \bar{a}^{\prime}$. Moving the elements of $D_{0} \cap\left(A \backslash \bar{a}^{0}\right)$ into $A^{\text {odd }}$ while keeping $C \cup M \cup \bar{a}^{0}$ fixed pointwise we obtain $F \subseteq C \cup M \cup A^{\text {odd }}$ such that for all $i$

$$
\text { (†) } \mathcal{N} \vDash \forall \bar{u}\left[\left\{\phi\left(b_{i}, \bar{a}, \bar{c}, \bar{u}\right) \& n(\bar{u})<s\right\} \Rightarrow\left(\exists \bar{v} \subseteq F \cup \bar{a} \bar{u} b_{i}\right) \neg \psi\left(b_{i}, \bar{a}, \bar{c}, \bar{v}\right)\right] .
$$

By deleting an initial segement of $\left\langle D_{i}: i<\omega\right\rangle$ we ensure that for all $i$

$$
\mathscr{N} \vDash\left(\forall \bar{v} \in F \cup \bar{a}^{\prime} b_{i} \bar{e}_{i}\right) \psi\left(b_{i}, \bar{a}, \bar{c}, \bar{v}\right) .
$$

By thinning $\left\langle D_{i}: i<\omega\right\rangle$ and permuting the entries of $\bar{u}$ we may suppose that $\bar{e}_{i}=\bar{g}_{i} \bar{h}_{i}$, where $\bar{g}_{i} \subseteq C \cup M \cup A^{\text {odd }}, \bar{h}_{i} \subseteq \bar{a}^{0}$ as sets, and $l\left(\bar{g}_{i}\right)$ and $l(\bar{h})_{i}=s$ do not depend on $i$. For each $i<\omega$ let $k(i)$ be the least $j \geq l\left(\bar{a}^{\prime}\right)$ such that $\bar{h}_{i} \subseteq\left\{a_{0}^{0}, \ldots, a_{j}^{0}\right\}$. Keeping $b_{i}$ and $\bar{g}_{i}$ fixed, rechoose $\bar{h}_{i}$ so as to minimize $k(i)$. Let $\bar{a}_{i}^{\prime}$ denote the initial segement of $\bar{a}^{i}$ of length $l\left(\bar{a}^{\prime}\right)$.

There are now two cases:

Case 1. $\operatorname{Sup}\{k(i): i<\omega\}<\omega$. By thinning the sequence $\left\langle D_{i}: i<\omega\right\rangle$ we may ensure that $\bar{h}_{i}=\bar{h}$ is fixed for all $i$. Let $\bar{h}_{j}$ stand in the same relation to $\bar{a}^{j}$ as $\bar{h}$ to $\bar{a}^{0}$. Since a map, which switches $\bar{a}^{0}$ and $\bar{a}^{2 j}$ for some $j \leq i$ and fixes the rest of $N$ pointwise, is an automorphism, from $(*)$ we have

$$
\mathscr{N} \vDash \phi\left(b_{i}, \bar{a}_{2 j}, \bar{c}, \bar{g}_{i} \bar{h}_{2 j}\right) \&\left(\forall \bar{v} \in D_{i} \cup b_{i} \bar{g}_{i} \bar{h}_{2 j}\right) \psi\left(b_{i}, \bar{a}_{2 j}, \bar{c}, \bar{v}\right) .
$$

By compactness there is a $\forall$-extension of $\mathscr{N}$ in which the formulas $\chi\left(y, \bar{a}_{2 j}, \bar{c}\right)$ $(j<\omega)$ have a common solution. This contradicts the almost disjointness property of $\chi$.

Case 2. Otherwise. In this case we obtain a contradiction to the stability of existential formulas. Let $\mathscr{N}^{\prime} \subseteq \mathscr{N}$ be the model defined by

$$
N^{\prime}=M \cup C \cup A^{\text {odd }} \cup F \cup\left\{a_{j}^{2 i}: i<\omega, j<2 i+l\left(\bar{a}^{\prime}\right)\right\} .
$$


Let $\bar{x}^{\prime}$ be a tuple of distinct variables extending $\bar{x}$ of length $l\left(\bar{a}^{\prime}\right)$. Let the formula

$$
\exists \bar{w}\left[\phi\left(b_{i}, \bar{x}, \bar{c}, \bar{g}_{i} \bar{w}\right) \&\left(\forall \bar{v} \in F \cup \bar{x}^{\prime} b_{i} \bar{g}_{i} \bar{w}\right) \psi\left(b_{i}, \bar{x}, \bar{c}, \bar{v}\right)\right]
$$

be denoted $\theta\left(\bar{x}^{\prime}, \bar{c}, b_{i}, \bar{g}_{i}\right)$. Since the universal quantifier is restricted to a specified finite set, this formula is equivalent to an existential one. Let $H_{i}$ denote $\left\{j: \mathscr{N}^{\prime} \vDash \theta\left(\bar{a}_{2 j}^{\prime}, \bar{c}, b_{i}, \bar{g}_{i}\right), j<\omega\right\}$. Let $\bar{h}_{i, j}$ denote the image of $\bar{h}_{i}$ under the map which takes $\bar{a}^{0}$ to $\bar{a}^{j}$. From (*) and (\#),

$$
\left.\mathscr{N} \vDash \phi\left(b_{i}, \bar{a}, \bar{c}, \bar{e}_{i}\right)\right) \&\left(\forall \bar{v} \in F \cup \bar{a} b_{i} \bar{e}_{i}\right) \psi\left(b_{i}, \bar{a}, \bar{c}, \bar{v}\right) .
$$

Switching $\bar{a}^{0}$ and $\bar{a}^{2 j}$ and fixing the rest of $N$ pointwise, we get

$$
\mathscr{N} \vDash \phi\left(b_{i}, \bar{a}_{2 j}, \bar{c}, \bar{g}_{i} \bar{h}_{i, 2 j}\right) \&\left(\forall \bar{v} \in F \cup \bar{a}_{2 j}^{\prime} b_{i} \bar{g}_{i} \bar{h}_{i, 2 j}\right) \psi\left(b_{i}, \bar{a}, \bar{c}, \bar{v}\right) .
$$

Since the set corresponding to $H_{i}$ in $\mathscr{N}$ is $\omega, H_{i}$ is the complement of some finite initial segement of $\omega$.

Towards a contradiction suppose $j \in \bigcap\left\{H_{i}: i<\omega\right\}$. For all $i<\omega$ we have $\bar{h}_{i}^{\prime} \in N^{\prime}$ such that

$$
\phi\left(b_{i}, \bar{a}_{2 j}, \bar{c}, \bar{g}_{i} \bar{h}_{i}^{\prime}\right) \&\left(\forall \bar{v} \in F \cup \bar{a}_{2 j}^{\prime} b_{i} \bar{g}_{i} \bar{h}_{i}^{\prime}\right) \psi\left(b_{i}, \bar{a}_{j}, \bar{c}, \bar{v}\right)
$$

is true in $\mathscr{N}^{\prime}$ and hence also in $\mathscr{N}$. In $\mathscr{N}$ we can switch $\bar{a}^{0}$ and $\bar{a}^{j}$, keeping the rest of the model fixed pointwise. This gives $\bar{h}_{i}^{*}$ such that

$(* *) \quad \mathcal{N} \vDash \phi\left(b_{i}, \bar{a}, \bar{c}, \bar{g}_{i} \bar{h}_{i}^{*}\right) \&\left(\forall \bar{v} \in F \cup \operatorname{Rng}\left(b_{i} \bar{g}_{i} \bar{h}_{i}^{*}\right) \psi\left(b_{i}, \bar{a}, \bar{c}, \bar{v}\right)\right)$.

From $(\dagger) n\left(\bar{g}_{i} \bar{h}_{i}^{*}\right)=s$, whence $\bar{h}_{i}^{*} \subseteq \bar{a}^{0}$. Since $\bar{a}^{2 j}$ is truncated in $\mathscr{N}^{\prime}$ and $\bar{h}_{i}^{*}$ is the image of $\bar{h}_{i} \subseteq \bar{a}^{2 j}$ on switching $\bar{a}^{0}$ and $\bar{a}^{2 j}$,

$$
\bar{h}_{i}^{*} \subseteq\left\{a_{0}^{0}, \ldots, a_{2 j+l\left(\bar{a}^{\prime}\right)}^{0}\right\} .
$$

Since $\left\{a_{0}^{0}, \ldots, a_{2 j+l\left(\bar{a}^{\prime}\right)}^{0}\right\} \subseteq D_{i}$ for all sufficiently large $i$, we have $\bar{h}_{i}^{*} \subseteq D_{i}$ for all sufficiently large $i$. From $(*)$ and $(* *)$ it follows that for all sufficiently large $i$

$$
\mathcal{N} \vDash \phi\left(b_{i}, \bar{a}, \bar{c}, \bar{g}_{i} \bar{h}_{i}^{*}\right) \&\left(\forall \bar{v} \in D_{i} \cup b_{i} \bar{g}_{i} \bar{h}_{i}^{*}\right) \psi\left(b_{i}, \bar{a}, \bar{c}, \bar{v}\right) .
$$

Thus, for all sufficiently large $i, \bar{h}_{i}^{*}$ is a possible choice for $\bar{h}_{i}$. Hence $k(i) \leq$ $2 j+l\left(\bar{a}^{\prime}\right)$ for all sufficiently large $i$, which contradicts the case hypothesis.

We have shown that $\bigcap\left\{H_{i}: i<\omega\right\}=\varnothing$. It follows easily that the existential formula $\theta(\bar{x}, \bar{z}, y, \bar{u})$ is unstable in $T$. This completes the proof of the Lemma 7.6.

7.7. Lemma. Let $\mathscr{M}$ be a model of a complete $\exists \forall$-theory. Let $A, C \subseteq M$ and $a \in M$. 
(1) The formula $x=y \vee \neg[x \downarrow y(D)]$ defines an equivalence relation on $M$.

(2) $[A \downarrow C \quad(D)] \Leftrightarrow(\forall a \in A)(\forall c \in C)[a \downarrow c \quad(D)]$.

(3) $a \downarrow a(D)$ if and only if $a \in \operatorname{acl}_{\mathscr{M}}(D)$ via some existential formula.

Proof. (1) Suppose that $\neg[a \downarrow b \quad(D)]$ and $\neg[b \downarrow c(D)]$. Towards a contradiction suppose that $a \downarrow c(D)$. From Lemma 7.6 there are two cases:

Case 1. $a \downarrow b \quad(\{c\} \cup D)$. From $a \downarrow c \quad(D)$ we have $a \downarrow\{c\} \cup D(D)$. By transitivity we have $a \downarrow\{b, c\} \quad(D)$, whence $a \downarrow b \quad(D)$, a contradiction.

Case 2. $c \downarrow b \quad(\{a\} \cup D)$. By the same argument switching $a$ and $c$ we obtain $b \downarrow c(D)$ which is again a contradiction.

(2) It is sufficient to prove that

$$
[A \cup\{b\} \downarrow C(D)] \Leftrightarrow[A \downarrow C(D) \& b \downarrow C(D)] .
$$

From left to right the implication is trivial. For the other direction suppose $A \downarrow C(D)$ and $b \downarrow C(D)$. From Lemma 7.6 there are two possibilities:

Case 1. $A \downarrow b \quad C \cup D)$. From $b \downarrow C \quad(D)$ we have $b \downarrow C \cup D(D)$. From $A \downarrow b$ $(C \cup D)$ we have $b \downarrow A \cup C \quad(C \cup D)$. From $b \downarrow C \cup D(D)$ and $b \downarrow A \cup C$ $(C \cup D)$ by transitivity we have $b \downarrow A \cup C(D)$. Hence, $b \downarrow C \quad(A \cup D)$, and so $C \downarrow A \cup\{b\} \quad(A \cup D)$. Also, $C \downarrow A \cup D(D)$ since $A \downarrow C \quad(D)$. From $C \downarrow A \cup D$ (D) and $C \downarrow A \cup\{b\} \quad(A \cup D)$ by transitivity we get $C \downarrow A \cup\{b\} \quad(D)$.

Case 2. $C \downarrow b \quad(A \cup D)$. In this case we get $C \downarrow A \cup\{b\} \quad(A \cup D)$ immediately. The rest of the argument is the same as in Case 1.

(3) $a \downarrow a(D)$ means that $a$ realizes the heir of $\operatorname{tp}(a \mid D)$ over $D \cup\{a\}$. This is the same as saying that, if $\mathscr{N} \supseteq_{\forall} \mathscr{M}$ is $\exists \forall$-saturated, then $a \in \operatorname{acl}_{\mathscr{N}}(D)$. From Theorem 4.1 this is the same as: there exist $n<\omega$ and an existential formula $\phi(x)$ over $D$ such that $\mathscr{N} \vDash \phi(a) \&\left(\exists^{\leq n} x\right) \phi(x)$. The desired conclusion is now clear.

Below the equivalence relation obtained in (1) of the last lemma will be denoted $E_{D}$.

7.8. Lemma. Let $\mathscr{M}$ be a model of a complete $\exists \forall$-theory, $a \in M, \alpha \in$ On, and $\left\langle A_{i}: i<\alpha\right\rangle$ be an increasing chain of subsets of $M$ such that

$$
\neg\left[A_{i+l} \downarrow a\left(A_{i}\right)\right] \text { for all } i<\alpha .
$$

Then $\alpha<|L|^{+}$.

Proof. Assume the hypothesis. Let $\Phi_{i}(x)=\exists: \forall-\operatorname{stp}\left(a \mid A_{i}\right) \quad(i<\alpha)$. From Lemma 7.5(a) for each $i<\alpha$ there exists a $\exists: \forall$-formula $\phi_{i}\left(x, \bar{y}_{i}\right)$ without parameters such that $\phi_{i}-\operatorname{Rk}\left(\Phi_{i+1}(x)\right)<\phi_{i}-\operatorname{Rk}\left(\Phi_{i}(x)\right)$. Since the value of the rank is a natural number, a particular $\exists: \forall$-formula of $L$ occurs at most a finite number of times in the sequence $\left\langle\phi_{i}(x, \bar{y}): i<\alpha\right\rangle$. Hence, $\alpha<|L|^{+}$as required. 


\section{MONADIC STABILITY AND TREE DECOMPOSITIONS}

In their paper [3] on second-order quantifiers and the complexity of theories Baldwin and Shelah introduced the notion of tree decomposability of theories. One of their results [3, Theorem 4.2.17] says that, if $T$ is stable and $\left(T_{\infty}\right.$, 2nd) $\Varangle(T$, Mon), then $T$ is tree-decomposable. Using $\exists: \forall$-stability instead of stability and similar, but not identical, reasoning we show that any complete $\exists \forall$-theory is tree-decomposable.

We begin by adapting four definitions from [3].

8.1.1. Definition. Let $\mathscr{M}$ be a structure for a relational language $A \subseteq M$, and $E$ be an equivalence relation on $M \backslash A$. Tuples $\bar{a}=\left(a_{1}, \ldots, a_{m}\right)$ and $\bar{b}=\left(b_{1}, \ldots, b_{n}\right)$ in $M \backslash A$ are called similar with respect to $E$ if $m=n$, $a_{i} E a_{j} \Leftrightarrow b_{i} E b_{j} \quad(1 \leq i, j \leq m)$, and

$$
\operatorname{tp}\left(a_{i_{1}} \cdots a_{i_{j}} \mid A\right)=\operatorname{tp}\left(b_{i_{1}} \cdots b_{i_{j}} \mid A\right)
$$

whenever $1 \leq i_{1}, \ldots, i_{j} \leq m$ and $a_{i_{1}}, \ldots, a_{i_{j}}$ are all in the same $E$-class. $E$ is called a congruence on $\mathscr{M}$ over $A$ if $\operatorname{tp}(\bar{a} \mid A)=\operatorname{tp}(\bar{b} \mid A)$ whenever $\bar{a}$, $\bar{b} \in M \backslash A$ are similar with respect to $E$.

8.1.2. Definition. $I$ is a tree if, for some $\lambda$ and $\kappa, I \subseteq \leq \kappa \lambda$ and

$$
\left[\sigma \in{ }^{\leq \kappa} \lambda \& \sigma \subseteq \tau \in I\right] \Rightarrow \sigma \in I .
$$

$\sigma$ is a tip of $I$ if $\sigma$ is maximal in $I$ with respect to $\subseteq$. The set of tips of $I$ is denoted $\mu(I)$. The height of $I$ is $\sup \{\operatorname{Dom}(\sigma)+1: \sigma \in I\}$.

8.1.3. Definition. The $L$-structure $\mathscr{M}$ is decomposed by the tree $I$ if there exist $L$-structures $\mathscr{M}_{\eta}, \mathscr{N}_{\eta}(\eta \in I)$ and equivalence relations $E_{\tau} \quad(\tau \in I \backslash \mu(I))$ such that for all $\eta, \rho \in I$ and $\tau \in I \backslash \mu(I)$ :

(i) $\left|N_{\eta}\right| \leq|L|+\aleph_{0}$.

(ii) If $\eta \subset \rho$, then $N_{\eta} \subset N_{\rho} \subseteq M_{\rho} \subseteq M_{\eta}$.

(iii) $E_{\tau}$ is a congruence on $\mathscr{M}_{\tau}$ over $N_{\tau}$. The $E_{\tau}$-classes are the sets $M_{\sigma} \backslash N_{\tau}$ such that $\sigma$ is a minimal extension of $\tau$ in $I$.

(iv) If $l(\eta)$ is a limit ordinal, then $N_{\eta}=\bigcup\left\{N_{\sigma}: \sigma \subset \eta\right\}$ and $M_{\eta}=$ $\bigcap\left\{M_{\sigma}: \sigma \subset \eta\right\}$.

(v) $M_{\langle\rangle}=M=\bigcup\left\{N_{\sigma}: \sigma \in I\right\}$.

8.1.4. Definition. $T$ is tree-decomposable if every model of $T$ is decomposed by some tree.

Our notion of $\mathscr{M}$ being decomposed by the tree $I$ is stronger in one respect than that of [3, Definition 3.2.1]. Since we require that $N_{\rho} \backslash N_{\eta} \neq \varnothing$ when $\eta \subset \rho$, the height of a tree decomposing $\mathscr{M}$ is at most $\left(|L|+\aleph_{0}\right)^{+}$. Since the same feature can conveniently be added to the definition just cited, this difference seems inessential. A more significant distinction lies in (iii) which is weaker here than in [3]. 
Let monadic logic be obtained by adjoining variables which range over subsets of the universe, and corresponding quantifiers, to the apparatus of first-order logic. We use $s, t, u$ possibly with superscripts and subscripts as set variables. There are new atomic formulas of the form $x \in s$.

8.1.5. Definition. The first-order theory $T$ is monadically unstable if there is a monadic formula $\phi(x, y, \bar{s})$ of the language of $T$ such that for every linear ordering $(I,<)$ of cardinality $>1$ there exist $\mathscr{M} \vDash T$ and $\bar{U}$, a sequence of subsets of $M$, such that $\phi(x, y, \bar{U})$ defines in $\mathscr{M}$ a linear ordering isomorphic to $(I,<)$ on the solution set of $\exists y[\phi(x, y, \bar{U}) \vee \phi(y, x, \bar{U})]$.

In $\S 8$ of [3] it is shown that the definition is equivalent if for $\phi(x, y, \bar{s})$ one reads $\phi(\bar{x}, \bar{y}, \bar{s})$, where $l(\bar{x})=l(\bar{y})=n . T$ is monadically stable if it is not monadically unstable. In the notation of [3] " $T$ monadically stable" is rendered " $(\mathrm{Th}(<)$, Mon $) \leq_{\text {Mon }}(T$, Mon $)$ ". From 3.2.4, 4.2.17, and 8.1.6 of [3] we observe:

$$
\begin{aligned}
& T \text { tree-decomposable } \\
& \quad \Rightarrow T \text { monadically stable } \\
& \quad \Rightarrow\left[T \text { stable } \&\left(T_{\infty}, 2 \text { nd }\right)\right. \\
& \quad \nless(T, \text { Mon })] \\
& \quad \Rightarrow T \text { tree-decomposable. }
\end{aligned}
$$

From the proof of Theorem 4.2.17 of [3] it is apparent that the last implication is true for our notion of tree-decomposable theory. Below we will verify that the first implication is also valid for our notion. It will then follow that for theories the two verisons of tree-decomposability are equivalent and the same as monadic stability. A point which emerges from the equivalence of tree-decomposability and monadic stability is that the choice of language for a theory makes no difference to whether the theory is tree-decomposable.

In order to show that tree-decomposability implies monadic stability we make a brief digression into a particular infinitary version of monadic logic, in the formulas of which the only free variables are those for subsets of the universe. When we apply this logic we shall have in mind a particular structure $\mathscr{M}$ and a particular subset $A$ of $M$. As initial formulas we take those of the form $\exists \bar{x} \Phi(\bar{x}, \bar{s})$, where $\bar{x}$ is a tuple of inidividual variables, $\bar{s}$ a tuple of set variables, and $\Phi(\bar{x}, \bar{s})$ a possibly infinite conjunction of atomic and negated atomic formulas over $A$. Other formulas are built up from initial formulas by negation, arbitrary conjunctions, and existential quantification of set variables. conjunctions are restricted to those having only a finite number of free variables. Formulas constructed in this way are called $L_{\infty, \omega}$ (Mon)-formulas over $A$. Note that every (finitary) monadic formula over $A$ which has no free individual variables is equivalent to an $L_{\infty, \omega}$ (Mon)-formula over $A$.

A set $\left\{\psi_{j}(\bar{S}): i \in J\right\}$ of $L_{\infty,(1)}$ (Mon)-formulas is a partitioning set if for every structure $\mathscr{M}$ such that $A \subseteq M$ and every tuple $\bar{U}$ of subsets of $M$ exactly one of the sentences $\psi_{j}(\bar{U})(j \in J)$ is true in $\mathscr{M}$. 
Let $\mathscr{M}$ be a structure, $A \subseteq M$, and $E$ be a congruence on $\mathscr{M}$ over $A$. Let $\bar{U}$ be a tuple of subsets of $M$. Let $\mathbb{C}(\mathscr{M}, E)$ denote the family of all substructures $\mathscr{N} \subseteq \mathscr{M}$ such that $A \subseteq N$ and $N \backslash A$ is an $E$-class. The next result is a simplified version of Lemma 3.1.13 of [3].

8.2. Lemma. Let a set $A$ and language $L$ be given. For every $L_{\infty, \omega}$ (Mon)formula $\phi(\bar{s})$ over $A$ there are a partitioning set $\left\{\psi_{j}(\bar{s}): j \in J\right\}$ of $L_{\infty, \omega}($ Mon)-formulas over $A$ and a cardinal $\lambda$ such that for every L-structure $\mathscr{M}$, with $M \supseteq A$, congruence relation $E$ on $\mathscr{M}$ over $A$, and tuples $\bar{U}_{0}, \bar{U}_{1}$ of subsets of $M$, we have

$$
\mathscr{M} \vDash \phi\left(\bar{U}_{0}\right) \leftrightarrow \phi\left(\bar{U}_{1}\right)
$$

whenever

$$
\begin{aligned}
\mid\{\mathscr{N} \in \mathbb{C}(\mathscr{M}, E): \mathscr{N} & \vDash \psi_{j}\left(\bar{U}_{0}\lceil N)\right\} \mid=\kappa \\
& \Leftrightarrow\left|\left\{\mathscr{N} \in \mathbb{C}(\mathscr{M}, E): \mathscr{N} \vDash \psi_{j}\left(\bar{U}_{1} \uparrow N\right)\right\}\right|=\kappa
\end{aligned}
$$

for all $j \in J$ and $\kappa<\lambda$.

Since the proof of Lemma 8.2 is routine we leave it to the reader.

8.3. Theorem. If $T$ is tree-decomposable, then $T$ is monadically stable.

Proof. Towards a contradiction let $T$ be tree-decomposable and monadically unstable.

Let $\phi(x, y, \bar{s})$ be a monadic formula representing all linear orderings of cardinality $>1$ in models of $T$. Choose a model $\mathscr{M}$ of $T$ and subsets $\bar{U}$ of $M$ such that $\phi(x, y, \bar{U})$ represents in $\mathscr{M}$ a cardinal $\gamma$. Let $D$ be the solution set of $\exists y[\phi(x, y, \bar{U}) \vee \phi(y, x, \bar{U})]$ in $\mathscr{M}$.

Let $\mathscr{M}$ be decomposed by the tree $I \subseteq{ }^{\leq \kappa} \lambda$ according to Definition 8.1.3. Recall that the height of the tree is at most $\left(|L|+\aleph_{0}\right)^{+}$. For any cardinal $\delta$ by setting $\gamma$ large enough we ensure that there exists $\tau \in I \backslash \mu(I)$ such that $D$ meets at least $\delta$ of the $E_{\tau}$-classes. Fix such $\tau$. for any $\sigma \subset \tau$ let $\sigma+$ denote the initial segment of $\tau$ of length $l(\sigma)+1$. We form $L^{*}$ by adjoining new unary relation symbols $R_{i}(i<l(\tau))$ to $L$, and we expand $\mathscr{M}$ to an $L^{*}$ structure $\mathscr{M}^{*}$ by letting $M_{\tau \uparrow i} \backslash M_{\tau \uparrow(i+1)}$ be the interpretation of $R_{i}$. Let $E$ be the equivalence relation on $M \backslash N_{\tau}$ defined by

$$
E=E_{\tau} \cup \bigcup\left\{E_{\sigma} \mid\left(M_{\sigma} \backslash M_{\sigma+}\right): \sigma \subset \tau\right\}
$$

Claim. $E$ is a congruence relation on $\mathscr{M}^{*}$ over $N_{\tau}$.

Proof of the Claim. Let $\sigma_{1} \subset \sigma_{2} \subset \cdots \subset \sigma_{n-1} \subset \tau$. Let $\bar{b}_{i j} \in M_{\sigma_{i}} \backslash M_{\left(\sigma_{i}\right)+}$ $\left(1 \leq i<n, 1 \leq j \leq k_{i}\right)$ and $\bar{b}_{n j} \in M_{\tau} \backslash N_{\tau} \quad\left(1 \leq j \leq k_{n}\right)$ be such that, for $1 \leq i \leq n$, any two entries of $\bar{b}_{i j}$ are $E$-related, and $\bar{b}_{i j}$ and $\bar{b}_{i j}$ fall in different $E$-classes whenever $j \neq j^{\prime}$. Note that, for $1 \leq i<n$, the $E$-class of $\bar{b}_{i j}$ is an $E_{\sigma_{i}}$-class, and the $E$-class of $\bar{b}_{n j}$ is an $E_{\tau}$-class. Let $\bar{c}_{i j} \in M \backslash N_{\tau}$ 
$\left(1 \leq i \leq n, 1 \leq j \leq k_{i}\right.$ ) be tuples falling in distinct $E$-classes such that $\operatorname{tp}\left(\bar{b}_{i j} \mid N_{\tau}\right)=\operatorname{tp}\left(\bar{c}_{i j} \mid N_{\tau}\right)$. To prove the Claim we have to show the concatenation of the $\bar{c}_{i j}$ realizes the same type as the concatenation of the $\bar{b}_{i j}$ over $N_{\tau}$. Let $\bar{a}_{i} \in N_{\sigma_{i}} \backslash N_{\sigma_{i-1}}(1<i<n)$, and $\bar{a}_{n} \in N_{\tau} \backslash N_{\sigma_{n-1}}$. It is sufficient to prove that the concatenation of the $\bar{a}_{i}$ and $\bar{c}_{i j}$ realizes the same type as the concatenation of the $\bar{a}_{i}$ and the $\bar{b}_{i j}$ over $N_{\sigma_{1}}$.

Because of the way in which $\mathscr{M}$ was expanded to $\mathscr{M}^{*}$ we see that $\bar{c}_{i j} \in$ $M_{\sigma_{i}} \backslash M_{\left(\sigma_{i}\right)+}(1 \leq i<n)$ and $\bar{c}_{n j} \in M_{\tau} \backslash N_{\tau}$. Since $E_{\tau}$ is a congruence relation on $\mathscr{M}_{\tau}$ over $N_{\tau}$, the concatenation $\bar{c}_{n}$ of the $\bar{c}_{n j} \quad\left(1 \leq j \leq k_{n}\right)$ realizes the same type over $N_{\tau}$ as the concatenation $\bar{b}_{n}$ of the $\bar{b}_{n j}$. Hence $\operatorname{tp}\left(\bar{c}_{n} \bar{a}_{n} \mid N_{\sigma_{n-1}}\right)=$ $\operatorname{tp}\left(\bar{b}_{n} \bar{a}_{n} \mid N_{\sigma_{n-1}}\right)$. For $1 \leq i<n$ let $\bar{c}_{i}$ denote the concatenation of the $\bar{c}_{i j}$ $\left(1 \leq j \leq k_{i}\right)$, and $\bar{b}_{i}$ the concatenation of the $\bar{b}_{i j} \quad\left(1 \leq i \leq k_{i}\right)$. By downward induction on $i$ we now see that

$$
\operatorname{tp}\left(\bar{c}_{n} \bar{a}_{n} \bar{c}_{n-1} \bar{a}_{n-1} \cdots \bar{a}_{i+1} \bar{c}_{i} \mid N_{\sigma_{i}}\right)=\operatorname{tp}\left(\bar{b}_{n} \bar{a}_{n} \bar{b}_{n-1} \bar{a}_{n-1} \cdots \bar{a}_{i+1} \bar{b}_{i} \mid N_{\sigma_{i}}\right)
$$

for $1 \leq i<n-1$. In the induction step we apply: $E_{\sigma_{i}}$ is a congruence relation on $M_{\sigma_{i}}$ over $N_{\sigma_{i}}, \bar{c}_{n} \bar{a}_{n} \bar{c}_{n-1} \bar{a}_{n-1} \cdots \bar{a}_{i+1}$ and $\bar{b}_{n} \bar{a}_{n} \bar{b}_{n-1} \bar{a}_{n-1} \cdots \bar{a}_{i+1}$ are in $M_{\left(\sigma_{i}\right)+}$ which is an $E_{\sigma_{i}}$-class disjoint from $\operatorname{Rng}\left(\bar{c}_{i}\right)$, and these tuples realize the same type over $N_{\sigma_{i}}$. Taking $i=1$ we see that $\bar{c}_{1} \cdots \bar{c}_{n} \bar{a}_{2} \cdots \bar{a}_{n}$ and $\bar{b}_{1} \cdots \bar{b}_{n} \bar{a}_{2} \cdots \bar{a}_{n}$ realize the same type over $N_{\sigma_{1}}$. This completes the proof of the Claim.

Let $\phi^{*}(t, u, \bar{s})$ be an $L_{\infty, \omega}($ Mon)-formula equivalent to the monadic formula

$$
\exists x \exists y[x \in t \& y \in u \& \phi(x, y, \bar{s})] .
$$

We are now apply Lemma 8.2 to the formula $\phi^{*}(t, u, \bar{s})$, the structure $\mathscr{M}^{*}$, and the congruence $E$. Let $\left\{\psi_{j}(t, u, \bar{s}): j \in J\right\}$ be the partitioning set of formulas and $\lambda$ the cardinal which result. It is crucial that $\delta$ should be larger than $|J|$. In this regard note that the partitioning set can be determined before we form $\mathscr{M}^{*}$. (We form $L^{*}$ by adjoining $|L|+\aleph_{0}$ new unary relation symbols to $L$. In expanding $\mathscr{M}$ to $\mathscr{M}^{*}$ we use as many of the new unary relations as we need, interpreting the rest as $\varnothing$. We also set aside $|L|+\aleph_{0}$ constants as names of the elements in $N_{\tau}$. If $\left|N_{\tau}\right|<|L|+\aleph_{0}$, some elements of $N_{\tau}$ have more than one name.) Let $a_{i} \in D(i<\delta)$ fall in different $E_{\tau}$-classes. For each $i<\delta$ let $M_{i}$ denote $N_{\tau} \cup\left(a_{i} / E\right)$ and $j_{i}$ the unique $j \in J$ such that $\mathscr{M}_{i}^{*} \vDash$ $\psi_{j}\left(\left\{a_{i}\right\}, \varnothing, \bar{U} \mid M_{i}\right)$. Since $|J|<\delta$, we can fix distinct $i, k<\delta$ such that $j_{i}=j_{k}$. It now follows from the conclusion of Lemma 8.2 that

$$
\left.\mathscr{M}^{*} \vDash \phi^{*}\left(\left\{a_{i}\right\},\left\{a_{k}\right\}, \bar{U}\right) \leftrightarrow \phi^{*}\left(\left\{a_{k}\right\},\left\{a_{i}\right\}, \bar{U}\right\}\right),
$$

whence $\mathscr{M} \vDash \phi\left(a_{i}, a_{k}, \bar{U}\right) \rightarrow \phi\left(a_{k}, a_{i}, \bar{U}\right)$. Since $a_{i} \neq a_{k}$, this contradicts the assumption that $\phi(x, y, \bar{U})$ defines a linear ordering in $\mathscr{M}$. This completes the proof of the theorem. 
In [3, Theorem 3.2.4] it was shown that stable theories which satisfy $\left(T_{\infty}\right.$, 2nd) $\Varangle(T$, Mon) are tree-decomposable. Using the $\exists: \forall$ stability theory worked out in the last section we now show that complete $\exists \forall$-theories are treedecomposable.

Let $\mathscr{M}, \mathscr{N}$ be structures with $\mathscr{M} \subseteq \mathscr{N} . A \subseteq M$ is called an a-subset of $\mathscr{M}$ with respect to $\mathscr{N}$ if for all $m, 1 \leq m<\omega$, and every $E \in F E_{\exists: \forall}^{m}(A)$ in the sense of $\mathcal{N}$ each $E$-class represented in $M^{m}$ is represented in $A^{m} . A \subseteq M$ is an a-subset of $\mathscr{M}$ if it is an $a$-subset of $\mathscr{M}$ with respect to $\mathscr{M}$.

In the next lemma $E_{A}$ denotes the equivalence relation on $N \backslash A$ defined by $x=y \vee \neg[x \downarrow y \quad(A)]$ in the sense of $\mathscr{N}$.

8.4. Lemma. Let $T$ be a complete $\exists \forall$-theory over the language $L$. Let $\mathscr{N} \vDash T$ and $\mathcal{N}^{\prime}$ be the expansion of $\mathcal{N}$ to $L^{\prime}$, where $L^{\prime}$ is formed from $L$ by adjoining a new relation symbol for each existential formula. Let $\mathscr{M}^{\prime} \subseteq \mathscr{N}^{\prime}, \mathscr{M}=\mathscr{M}^{\prime} \uparrow L$, $A \subseteq M$ be an a-subset of $\mathscr{M}$ with respect to $\mathscr{N}$, and $E$ be the restriction of $E_{A}$ to $M \backslash A$. Then $E$ is a congruence on $\mathscr{M}^{\prime}$ over $A$.

Proof. It is sufficient to prove that, if $\bar{a}_{0}, \bar{a}_{1}, \bar{b}_{0}, \bar{b}_{1} \in M \backslash A, l\left(\bar{a}_{i}\right)=l\left(\bar{b}_{i}\right)$ $(i<2)$,

$$
\left(\operatorname{Rng}\left(\bar{a}_{0}\right) / E\right) \cap\left(\operatorname{Rng}\left(\bar{a}_{1}\right) / E\right)=\left(\operatorname{Rng}\left(\bar{a}_{0}\right) / E\right) \cap\left(\operatorname{Rng}\left(\bar{a}_{1}\right) / E\right)=\varnothing,
$$

and $\operatorname{tp}_{\mathscr{N}^{\prime}}\left(\bar{a}_{i} \mid A\right)=\operatorname{tp}_{\mathscr{N}^{\prime}}\left(\bar{b}_{i} \mid A\right) \quad(i<2)$, then $\operatorname{tp}_{\mathscr{N}^{\prime}}\left(\bar{a}_{0} \bar{a}_{1} \mid A\right)=\operatorname{tp}_{\mathscr{N}^{\prime}}\left(\bar{b}_{0} \bar{b}_{1} \mid A\right)$. In $\mathscr{N}$ we have $\exists: \forall-\operatorname{tp}\left(\bar{a}_{i} \mid A\right)=\exists: \forall-\operatorname{tp}\left(\bar{b}_{i} \mid A\right) \quad(i<2)$, and from Lemma 7.7(2) $\operatorname{Rng}\left(\bar{a}_{0}\right) \downarrow \operatorname{Rng}\left(\bar{a}_{1}\right) \quad(A)$ and $\operatorname{Rng}\left(\bar{b}_{o}\right) \downarrow \operatorname{Rng}\left(\bar{b}_{1}\right) \quad(A)$. Since $A$ is an $a$-subset of $\mathscr{M}$ with respect to $\mathscr{N}$,

$$
\exists: \forall-\operatorname{stp}_{\mathscr{N}}\left(\bar{a}_{i} \mid A\right)=\exists: \forall-\operatorname{stp}_{\mathscr{N}}\left(\bar{b}_{i} \mid A\right) \quad(i<2) .
$$

Together with the independence noted above, this gives

$$
\exists: \forall-\operatorname{stp}_{\mathscr{N}}\left(\bar{a}_{0} \bar{a}_{1} \mid A\right)=\exists: \forall-\operatorname{stp}_{\mathscr{N}}\left(\bar{b}_{0} \bar{b}_{1} \mid A\right) .
$$

Hence, $\operatorname{tp}_{\mathscr{M}^{\prime}}\left(\bar{a}_{0} \bar{a}_{1} \mid A\right)=\operatorname{tp}_{\mathscr{M}^{\prime}}\left(\bar{b}_{0} \bar{b}_{1} \mid A\right)$ as required.

8.5. Theorem. If $T$ is a complete $\exists \forall$-theory, then $T$ is tree-decomposable.

Proof. Let $L^{\prime}$ be the language obtained from the language $L$ of $T$ by adjoining a new relation symbol for each existential formula of $L$. Let $T^{\prime}$ be the $L^{\prime}$ theory corresponding to $T$. Let $\mathscr{M}^{\prime} \vDash T^{\prime}$ and $\mathscr{M}=\mathscr{M}^{\prime} \mid L$.

We establish a tree decomposition of $\mathscr{M}^{\prime}$ as follows. Let $\mathscr{N}_{\langle\rangle}$be a substructure of $\mathscr{M}$ of cardinality $\left|L^{\prime}\right|$ such that $N_{\langle\rangle}$is an a-subset of $\mathscr{M}$. Let $\mathscr{M}_{\langle\rangle}^{\prime}$ be $\mathscr{M}^{\prime}$. Enumerate \langle\rangle in the tree $I$.

Suppose that $\mathscr{N}_{\eta}$ and $\mathscr{M}_{\eta}^{\prime}$ have been defined such $\mathscr{N}_{\langle\rangle} \subseteq \mathscr{N}_{\eta} \subset \mathscr{M}_{\eta}^{\prime} \subseteq \mathscr{M}^{\prime}$ and $N_{\eta}$ is an a-subset of $\mathscr{M}_{\eta}=\mathscr{M}_{\eta}^{\prime} \uparrow L$ with respect to $\mathscr{M}$, where $\eta \in I$ is a sequence of ordinals of length $\left\langle\left|L^{\prime}\right|^{+}\right.$. Let $E_{\eta}$ denote the restriction of $E_{N_{\eta}}$ (relative to $\left.\mathscr{M}\right)$ [Bto $M_{\eta} \backslash N_{\eta}$. By the previous lemma $E_{\eta}$ is a congruence on $\mathscr{M}_{\eta}^{\prime}$ over $N_{\eta}$. Let $\left\{C_{i}: i<\alpha_{\eta}\right\}$ be an enumeration of the $E_{\eta}$-classes. 
Enumerate $\eta^{\wedge}\langle i\rangle$ in the tree $I$ for each $i<\alpha_{\eta}$. Define $\mathscr{M}_{\eta}^{\prime}{ }^{\prime}\langle i\rangle$ to be the substructure of $\mathscr{M}^{\prime}$ with universe $N_{\eta} \cup C_{i} \quad\left(i<\alpha_{\eta}\right)$. Let $N_{\eta \hat{\eta}\langle i\rangle}$ be an $a$-subset of $\mathscr{M}_{\eta^{\hat{\langle}}\langle i\rangle}$ with respect to $\mathscr{M}$ such that $N_{\eta} \subset N_{\hat{\eta}^{\hat{\beta}}\langle i\rangle}$ and $\left|N_{\eta^{\hat{\langle}}\langle i\rangle}\right| \leq\left|L^{\prime}\right|_{0}$. We continue the construction as far as possible. If $\xi$ is a sequence of ordinals of limit length $<\left|L^{\prime}\right|^{+}$such that $\eta \in I$ for all $\eta \subset \xi$, we define

$$
N_{\xi}=\bigcup\left\{N_{\eta}: \eta \subset \xi\right\}, \quad M_{\xi}=\bigcap\left\{M_{\eta}: \eta \subset \xi\right\} .
$$

Note that $N_{\xi}$ is an $a$-subset of $\mathscr{M}_{\xi}$ with respect to $\mathscr{M}$. If $\mathscr{N}_{\eta}=\mathscr{M}_{\eta}^{\prime}$, then $\eta$ is a tip of the tree. (Branches of length $\left|L^{\prime}\right|^{+}$are not ruled out however.)

We claim that $M=\bigcup\left\{N_{\eta}: \eta \in I\right\}$. Towards a contradiction suppose $a \in$ $M \backslash \bigcup\left\{N_{\eta}: \eta \in I\right\}$. Let $\xi=\bigcup\left\{\eta \in I: a \in M_{\eta}\right\}$. Since $a \in M_{\eta} \backslash N_{\eta}$ for all $\eta \subset \xi, l(\xi)=\left|L^{\prime}\right|^{+}$. For all $j<\left|L^{\prime}\right|^{+}$let $A_{j}=N_{\xi \mid j}$. Consider particular $j<\left|L^{\prime}\right|^{+}$and let $\eta=\xi \mid j$. Let $\eta^{\wedge}\langle i\rangle \subseteq \xi$. Then $M_{\eta^{\wedge}\langle i\rangle}=N_{\eta} \cup C_{i}=A_{j} \cup C_{i}$. Moreover, $A_{j} \subset A_{j+1} \subseteq A_{j} \cup C_{i}$. Since $C_{i}$ is included in an $E_{A_{j}}$-class $a \in C_{i}$, and $A_{j+1} \cap C_{i} \neq \varnothing$, we have $\neg\left[A_{j+1} \downarrow a\left(A_{j}\right)\right]$ by Lemma 7.7. It follows by Lemma 7.8 that the range of $j$ has cardinality $<\left|L^{\prime}\right|^{+}$. This contradiction confirms that the tree decomposition exhausts $\mathscr{M}^{\prime}$ and that the tree $I$ has ordinal height $\leq\left|L^{\prime}\right|^{+}$.

We have shown that $T^{\prime}$ is tree-decomposable. As we observed in the discussion following Definition 8.1.5 a theory which is tree-decomposable with respect to one language is tree-decomposable with respect to every language. Hence, $T$ is also tree-decomposable.

8.6. Corollary. Complete $\exists \forall$-theories are monadically stable. A fortiori, such theories are stable.

\section{Prime MOdels}

In this section we confine consideration to finite relational languages. On one hand, we give an example to show that not all complete $\exists \forall$-theories have prime models over sets; on the other hand we show that each such theory has a prime model over $\varnothing$.

Recall Example 1 from the introduction to Part I. Let $\mathscr{M}^{*}=\dot{\bigcup}_{i<\omega} \mathscr{M}_{i}$ be a disjoint union of finite trees such that every isomorphism type of finite trees is represented by some $\mathscr{M}_{i}$. By "tree" here we mean a graph with no cycles. The complete $\exists \forall$-theory we have in mind is $T=\operatorname{Th}\left(\mathscr{M}^{*}\right)$. Let $R$ be the binary relation symbol which constitutes the language of graphs. Let $\mathscr{N}$ be a tree disjoint from $\mathscr{M}^{*}$ with universe

$$
\{\varnothing\} \cup\{X \subseteq \omega:|X|=\omega\} \cup\{(X, n, i): i \leq n, n \in X \subseteq \omega,|X|=\omega\} .
$$

Let $R^{N}$ be the symmetric closure of

$$
\begin{gathered}
\{(\varnothing, X): X \subseteq \omega,|X|=\omega \mid\} \cup\{(X,(X, n, 0)): n \in X \subseteq \omega,|X|=\omega\} \\
\cup\{(X, n, i),(X, n, i+1): i<n, n \in X \subseteq \omega,|X|=\omega\} .
\end{gathered}
$$


Consider the model $\mathscr{M}=\mathscr{M}^{*} \cup \mathscr{N}$ of $T$, and let $a$ denote $\varnothing \in N$.

Claim. There is no prime model over $\{a\}$.

Proof. Let $T_{0}$ denote $\operatorname{Th}(\mathscr{M}, a)$, the theory obtained by naming $a$. Let $\mathscr{M} \backslash\{a\}$ denote the graph obtained from $\mathscr{M}$ by deleting $a$. Let $\pi_{n}(x, y)$ be a formula such that $\mathscr{M} \vDash \pi_{n}(a, b)$ means that $b$ is a neighbor of $a$, and for every $c \in M$, if there is a path of length $n+1$ from $b$ to $c$ in $\mathscr{M} \backslash\{a\}$, then $c$ has at most two neighbors. Let $\sigma_{n}(x, y), \tau_{n}(x, y)$ be formulas such that $\mathscr{M} \vDash \sigma_{n}(a, b)$ means that there is a unique $c \in M$ with only one neighbour which lies at distance $n+1$ from $b$ in $\mathscr{M} \backslash\{a\}$, and $\mathscr{M} \vDash \pi_{n}(a, b)$ means there is no $c \in M$ with only one neighbor which lies at distance $n+1$ from $b$ in $\mathscr{M} \backslash\{a\}$. For $X \subseteq \omega$ let $\Sigma_{X}(x)$ denote the set

$$
\left\{\pi_{n}(a, x): n<\omega\right\} \cup\left\{\sigma_{n}(a, x): n \in X\right\} \cup\left\{\tau_{n}(a, x): n<\omega, n \notin X\right\} .
$$

The crucial point is that, if $|X|=\omega$, then $\Sigma_{X}(x)$ generates $\operatorname{Tp}_{\mathscr{M}}\left(b_{X} \mid a\right)$, where $b_{X}$ denotes $X$ seen as a member of $M$. If $\Sigma \subseteq \Sigma_{X}$ is finite, then there exists infinite $Y \subseteq \omega$ such that $\Sigma \subseteq \Sigma_{Y}$ and $Y \neq X$. Clearly, $\Sigma_{X} \cup \Sigma_{Y}$ is inconsistent. Therefore no neighbor $b$ of $a$ in $M$ realizes an isolated type over $\{a\}$. This completes the proof of the claim.

We will now show that every complete $\exists \forall$-theory over a finite language has a prime model. Before proving this we need some lemmas about rank. For the rest of this section $T$ denotes a complete $\exists \forall$-theory over a finite relational language and $\vdash \theta$ means that $\theta$ is valid in every model of $T$. A formula $\chi(\bar{y})$ is called a $\forall \cap \exists$-formula if in $T$ it is equivalent to a $\forall$-formula and to a $\exists$-formula; $\phi(\bar{x})$ is called almost quantifier-free if it is $\forall \cap \exists$ and equivalent to some quantifier-free formula over $M$ for each $\mathscr{M} \vDash T$.

9.1. Lemma. Let $\phi(\bar{x})$ be almost quantifier-free in $T, \operatorname{Rk}(\phi(\bar{x}))=r$, and $\theta(\bar{x}, \bar{y})$ be quantifier-free. There exists a $\forall \cap \exists$-formula $\chi(\bar{y})$ such that for all $\mathscr{M} \vDash T$ and $\bar{b} \subseteq M, R(\phi(\bar{x}) \& \theta(\bar{x}, \bar{b}))=r$ iff $\mathscr{M} \vDash \chi(\bar{b})$.

This is essentially the same as Lemma 3.1 of [9] so we omit the proof.

9.2. Lemma. Let $\phi(\bar{x})$ be a formula which in $T$ is nonnull and almost quantifier-free. Let $\operatorname{Rk}(\phi(\bar{x}))=r$. There exists a nonnull $\forall$-formula $\psi(\bar{x})$ such that for every $\mathscr{M} \vDash T$ and all $\bar{a} \subseteq M$,

$$
\mathscr{M} \vDash \psi(\bar{a}) \leftrightarrow[\phi(\bar{a}) \& \operatorname{Rk}(\operatorname{tp}(\bar{a} \mid M \backslash \bar{a}))=r] .
$$

Proof. Consider $\mathscr{M} \vDash T$ and $\bar{a} \subseteq M$ such that $\mathscr{M} \vDash \phi(\bar{a})$. If $\operatorname{Rk}(\operatorname{tp}(\bar{a} \mid M \backslash \bar{a}))$ $<r$, then there exist a quantifier-free formula $\theta(\bar{x}, \bar{y})$ and $\bar{b} \subseteq M \backslash \bar{a}$ such that $\mathscr{M} \vDash \theta(\bar{a}, \bar{b})$ and $\operatorname{Rk}(\phi(\bar{x}) \& \theta(\bar{x}, \bar{b}))<r$. Moreover, such $\theta(\bar{x}, \bar{y})$ may be found which is the intersection of at most $\operatorname{Mt}(\phi(\bar{x}))$ basic formulas. Now fix a quantifier-free formula $\theta(\bar{x}, \bar{y})$. From the last lemma there exists a $\forall \cap \exists-$ formula $\chi_{\theta}(\bar{y})$ such that for any $\mathscr{M} \vDash T$ and $\bar{a} \subseteq M$

$$
\mathscr{M} \vDash \forall \bar{y}\left[\bar{y} \cap \bar{a}=\varnothing \& \theta(\bar{a}, \bar{y}) . \rightarrow \chi_{\theta}(\bar{y})\right]
$$


iff no instance of $\theta(\bar{x}, \bar{y})$ witnesses $\operatorname{Rk}(\operatorname{tp}(\bar{a} \mid M \backslash \bar{a}))<r$. Since $L(T)$ is finite there are at most a finite number of formulas $\theta(\bar{x}, \bar{y})$ which are the intersection of at most $\operatorname{Mt}(\phi(\bar{x}))$ basic formulas. Taking the conjunction of $\phi(\bar{x})$ and of $\forall \bar{y}\left[\bar{y} \cap \bar{x}=\varnothing \& \theta(\bar{x}, \bar{y}) . \rightarrow \chi_{\theta}(\bar{y})\right]$ over all such $\theta$ we obtain the desired formula $\psi(\bar{x})$.

It remains to show that $\psi(\bar{x})$ is nonnull. It is easy to find models $\mathscr{M}, \mathscr{N}$ of $T$ and $\bar{a} \in N$ such that $\mathscr{M} \succeq \mathscr{N} \vDash \phi(\bar{a})$ and $\operatorname{Rk}\left(\operatorname{tp}_{\mathscr{N}}(\bar{a} \mid M)\right)=r$. Let $\mathscr{M} \cup \bar{a}$ denote the substructure of $\mathscr{N}$ with universe $M \cup \bar{a}$. By Lemma 0.2, for any formula $\chi(\bar{x})$ over $M$

$$
\mathrm{R}_{\mathscr{M}}(\chi(\bar{x})) \leq \mathrm{Rk}_{\mathscr{M} \cup \bar{a}}(\chi(\bar{x})) \leq \mathbf{R}_{\mathscr{N}}(\chi(\bar{x})),
$$

and $\mathrm{Rk}_{\mathscr{M}}(\chi(\bar{x}))=\mathrm{Rk}_{\mathscr{N}}(\chi(\bar{x}))$ since $\mathscr{M} \preceq \mathscr{N}$. Hence,

$$
\mathrm{Rk}_{\mathscr{M} \cup \bar{a}}(\chi(\bar{x}))=\mathrm{Rk}_{\mathscr{M}}(\chi(\bar{x}))
$$

which means that $\operatorname{Rk}_{\mathscr{M} \cup \bar{a}}(\operatorname{tp}(\bar{a} \mid M \backslash \bar{a}))=r$. Thus, $\psi(\bar{x})$ has a solution in $\mathscr{M} \cup \bar{a}$ which suffices since $\mathscr{M} \cup \bar{a} \vDash T$. This completes the proof.

The formula $\phi(\bar{x})$ is totally nonalgebraic in $T$ if

$$
\vdash \phi(\bar{x}) \rightarrow \bigwedge\left\{x_{i} \notin \operatorname{acl}(\varnothing): 1 \leq i \leq n\right\},
$$

where $\bar{x}=\left(x_{1}, \ldots, x_{n}\right)$.

A formula $\psi(\bar{x})$ is called $T$-complete if it isolates an elementary $l(\bar{x})$-type over $\varnothing$.

Let $\psi(\bar{x})$ be a $\forall$-formula which is $T$-complete. From $\psi(\bar{x})$ we construct an extension $T^{\prime}=T[\psi(\bar{x})]$ of $T$ as follows. From $L\left(T^{\prime}\right)$ by adjoining new unary relation symbols, one for each entry in $\bar{x}$. Let $\rho(\bar{x})$ denote the conjunction of all atomic formulas $R(u)$, where $u$ is an entry of $\bar{x}$ and $R$ is the corresponding unary relation symbol. The axioms of $T^{\prime}$ are those of $T$, universal and existential sentences saying that the interpretation of each of the new relation symbols is a singleton, and $\forall \bar{x}[\rho(\bar{x}) \rightarrow \psi(\bar{x})]$. Notice that $T[\psi(\bar{x})]$ is a complete $\exists \forall$-theory. Every model $\mathscr{M}$ of $T$ can be expanded to a model $\mathscr{M}[\bar{a}]$ of $T^{\prime}$ by picking a solution $\bar{a}=\left(a_{1}, \ldots, a_{l(\bar{a})}\right)$ of $\psi(\bar{x})$ in $\mathscr{M}$ and letting $a_{i}$ be the unique solution of $R$, the unary predicate symbol corresponding to $x_{i}$. Clearly every model of $T^{\prime}$ arises in this way.

For each formula $\theta(\bar{y})$ of $T^{\prime}$ in which no entry of $\bar{x}$ occurs, denote by $\theta^{\#}(\bar{x}, \bar{y})$ the formula obtained from $\theta(\bar{y})$ by substituting for each atomic formula $R(u)$, with $R$ one of the new unary predicates, the formula $x_{i}=u$, where $x_{i}$ is the entry of $\bar{x}$ corresponding to $R$. Denote by $\theta_{T}(\bar{x}, \bar{y})$ the formula $\psi(\bar{x}) \& \theta^{\#}(\bar{x}, \bar{y})$. Observe that, if $\vdash_{T^{\prime}} \theta(\bar{y}) \rightarrow \chi(\bar{y})$, where $\chi(\bar{y})$ is a formula of $T$, then $\vdash \theta_{T}(\bar{x}, \bar{y}) \rightarrow \chi(\bar{y})$. Further, if $\theta(\bar{y})$ is $T^{\prime}$-complete, then $\theta_{T}(\bar{x}, \bar{y})$ is $T$-complete.

9.3. Lemma. Let $\phi(\bar{x})$ be a formula of $T$ which is nonnull and almost quantifierfree. There is a T-complete $\forall$-formula $\psi(\bar{x})$ which implies $\phi(\bar{x})$.

Proof. We need the following claims: 
Claim 1. Let $\mathscr{M} \vDash T$ and $\bar{a} \in \operatorname{acl}_{\mathscr{M}}(\varnothing) . \operatorname{Tp}(\bar{a})$ is isolated by a $\forall \cap \exists$-formula.

Proof of Claim 1. Since it makes no difference we assume $l(\bar{a})=1$ and write $a$ for $\bar{a}$. From Lemma 4.1, $a$ is the solution of a $\exists$-formula which has only a finite number of solutions. Let $\psi(x)$ be a $\exists$-formula such that $\mathscr{M} \vDash \psi(a)$ and $\psi(x)$ has as few solutions as possible. By Lemma 3.1, $T$ has a $\exists \forall$-saturated model $\mathscr{N}$. From Corollary 3.9 two elements realize the same elementary type in $\mathscr{N}$ iff they realize the same $\exists$-type. Thus, if $\psi(x)$ does not isolate $\operatorname{Tp}(a)$, there is an existential formula $\theta(x)$ such that $\psi(x) \& \theta(x)$ is satisfiable but $\mathscr{M} \vDash$ $\neg \theta(a)$. Now there is a $\exists$-formula equivalent to $\psi(x) \& \exists y[\psi(y) \& \theta(y) \& x \neq y]$; such a formula contradicts the choice of $\psi(x)$ since it has fewer solutions. Thus, $\psi(x)$ isolates the type of $a$. Suppose that $\psi(x)$ has $m$ solutions. Then

$$
\forall y_{1} \cdots \forall y_{m}\left[\bigwedge_{1 \leq i<j \leq m} y_{i} \neq y_{j} \& \bigwedge_{1 \leq i \leq m} \psi\left(y_{i}\right) \cdot \rightarrow \cdot \bigvee_{1 \leq i \leq m} x=y_{i}\right]
$$

also isolates the type of $a$ and is equivalent to a $\forall$-formula. This completes the proof of the claim.

Claim 2. In the statement of the lemma we can suppose that $\phi(\bar{x})$ is totally nonalgebraic.

Proof of the Claim 2. In proving the lemma we can proceed by induction on $l(\bar{x})$. Suppose $\phi(\bar{x})$ has a solution $\bar{a}$ in a model $\mathscr{M}$ of $T$ in which one of the entries is algebraic. Let $\bar{x}=x \bar{x}^{\prime}$ and $\bar{a}=a \bar{a}^{\prime}$ with $a \in \operatorname{acl}(\varnothing)$. Let $\theta(x)$ be a $\forall$-formula isolating $\operatorname{Tp}(a), T^{\prime}=T[\theta(x)]$, and $R$ denote the new unary relation symbol $R$. Let $\mathscr{M}^{\prime}=\mathscr{M}[a]$ be the model of $T^{\prime}$ obtained from $\mathscr{M}$ by letting $\{a\}$ be the interpretation of $R$. Clearly, $T^{\prime}$ is a complete $\exists \forall$-theory. Let $\phi^{\prime}(\bar{x})$ be the formula $\exists x\left[R(x) \& \phi\left(x, \bar{x}^{\prime}\right)\right]$ which is equivalent in $T^{\prime}$ to $\forall x\left[R(x) \& \phi\left(x, \bar{x}^{\prime}\right)\right]$. Clearly, $\mathscr{M}^{\prime} \vDash \phi^{\prime}\left(\bar{a}^{\prime}\right)$ and $\phi^{\prime}\left(\bar{x}^{\prime}\right)$ is almost quantifier-free. By the induction hypothesis there is a $T^{\prime}$-complete $\forall$-formula $\psi^{\prime}\left(\bar{x}^{\prime}\right)$ which implies $\phi^{\prime}\left(\bar{x}^{\prime}\right)$ in $T^{\prime}$. Without loss of generality, $x$ does not occur in $\psi^{\prime}\left(\bar{x}^{\prime}\right)$. Let $\psi(\bar{x})$ be a $\forall$-formula equivalent to $\left(\psi^{\prime}\right)_{T}(\bar{x})$. Then $\psi(\bar{x})$ satisfies the conclusion of the lemma. This completes the proof of the claim.

We now recall an idea used in [9, Definition 3.1]. Fix the formula $\phi(\bar{x})$ which is now assumed to be totally nonalgebraic. Let $(\mathbf{R k}, \mathbf{M t})(\phi(\bar{x}))=(r, m)$. Consider a $\exists \forall$-saturated model $\mathscr{M}$ of $T$. A tuple $\bar{a} \subseteq M$ is called independent if $\mathscr{M} \vDash \phi(\bar{a})$ and $\operatorname{Rk}(\phi(\bar{x}) \& \theta(\bar{x}, \bar{b}))=r$ for every quantifier-free formula $\theta(\bar{x}, \bar{b})$ such that $\bar{b} \subseteq M \backslash \bar{a}$ and $\mathscr{M} \vDash \phi(\bar{a}) \& \theta(\bar{a}, \bar{b})$. Let $I(\mathscr{M})$ denote the set of all independent tuples $\bar{a} \subseteq M$. Define the binary relation $E$ on $I(\mathscr{M})$ by: $\bar{a} E \bar{a}^{\prime}$ if $\mathscr{M} \vDash \theta(\bar{a}, \bar{b}) \leftrightarrow \theta\left(\bar{a}^{\prime}, \bar{b}\right)$ for every atomic formula $\theta(\bar{x}, \bar{y})$ and all $\bar{b} \subseteq M \backslash\left(\bar{a} \cup \bar{a}^{\prime}\right)$.

The next claim corresponds to Proposition 3.2 of [9].

Claim 3. (1) $I(\mathscr{M})$ is definable by a $\forall$-formula.

(2) $I(\mathscr{M}) \neq \varnothing$. 
(3) $E$ is definable by a $\forall$-formula.

(4) For all $\bar{a} \in I(\mathscr{M})$ and $B \subseteq M$ there exists $\bar{a}^{\prime}$ such that $\bar{a} E \bar{a}^{\prime}, \operatorname{Tp}(\bar{a})=$ $\operatorname{Tp}\left(\bar{a}^{\prime}\right)$, and $\bar{a}^{\prime} \cap B=\varnothing$.

(5) $E$ is an equivalence relation with $m$ classes.

(6) If $\bar{a}_{0} E \bar{a}_{1}$, then $\operatorname{Tp}\left(\bar{a}_{0}\right)=\operatorname{Tp}\left(\bar{a}_{1}\right)$.

Proof of Claim 3. (1) and (2) follow from the previous lemma and (3) is immediate since the language is finite and relational.

(4) First notice that the conclusion holds if we drop the requirement that $\bar{a} E \bar{a}^{\prime}$. Otherwise there are $\bar{a} \in I(\mathscr{M})$ and a finite set $B \subseteq M$ such that for all $\bar{a}^{\prime} \subseteq M$

$$
\operatorname{Tp}\left(\bar{a}^{\prime}\right)=\operatorname{Tp}(\bar{a}) \rightarrow \bar{a}^{\prime} \cap B \neq \varnothing .
$$

Since $\mathscr{M}$ is $\exists \forall$-saturated, and elementary types are determined by $\exists$-types in $\mathscr{M}$, there exists $\theta(\bar{x})$ a conjunction of a $\exists$-formula and a $\forall$-formula such that $\theta(\bar{x}) \in \operatorname{Tp}(\bar{a})$ and

$$
\mathscr{M} \vDash \forall \bar{x}[\theta(\bar{x}) \rightarrow \bar{x} \cap B \neq \varnothing] .
$$

From Sublemma 5.2.1 such $B$ can be found $\subseteq \operatorname{acl}(\varnothing)$. This contradicts the fact that $\phi(\bar{x})$ is totally nonalgebraic.

Now we can choose $\bar{a}_{i} \subseteq M(i<\omega)$ such that $\operatorname{Tp}\left(\bar{a}_{i}\right)=\operatorname{Tp}(\bar{a})$ and $\bar{a}_{i} \cap \bar{a}_{j}=$ $\varnothing(i<j<\omega)$. By Ramsey's Theorem the $\bar{a}_{i}$ can be chosen such that either $\bar{a}_{i} E \bar{a}_{j} \quad(i<j<\omega)$ or $\neg\left(\bar{a}_{i} E \bar{a}_{j}\right) \quad(i<j<\omega)$. In the former case $\bar{a}_{0}$ clearly has the property desired for $\bar{a}$. Since $\mathscr{M}$ is $\aleph_{0}$-homogeneous by Lemma 3.2 and $\operatorname{Tp}(\bar{a})=\operatorname{Tp}\left(\bar{a}_{o}\right), \bar{a}$ also has the property. It remains to consider the case in which $\neg\left(\bar{a}_{i} E \bar{a}_{j}\right) \quad(i<j<\omega)$. From the definition of $E$ for each pair $(i, j)$ wiht $i<j<\omega$ there exists a basic formula $\theta_{i j}\left(\bar{x}, \bar{b}_{i j}\right)$ such that $\bar{b}_{i j} \cap\left(\bar{a}_{i} \cup \bar{a}_{j}\right)=\varnothing$ and $\mathscr{M} \vDash \theta_{i j}\left(\bar{a}_{i}, \bar{b}_{i j}\right) \& \neg \theta_{i j}\left(\bar{a}_{j}, \bar{b}_{i j}\right)$. By another application of Ramsey's Theorem we can suppose that $\bar{b}_{i j} \cap \bar{a}_{k}=\varnothing \quad(i<j<\omega, k<\omega)$. Let $D$ denote $\bigcup\left\{\bar{b}_{i j}: i<j \leq m\right\}$. Then $\bar{a}_{0}, \ldots, \bar{a}_{m}$ realize distinct types of rank $r$ over $D$. This contradicts $\operatorname{Mt}(\phi(\bar{x}))=m$.

(5) We wish to show that $E$ is an equivalence relation. Towards a contradiction suppose that $\bar{a}_{0} E \bar{a}_{1}, \bar{a}_{1} E \bar{a}_{2}$, and $\neg \bar{a}_{0} E \bar{a}_{2}$. There exists a basic formula $\theta(\bar{x}, \bar{b})$ such that $\bar{b} \cap\left(\bar{a}_{0} \cup \bar{a}_{2}\right)=\varnothing$ and $\mathscr{M} \vDash \theta\left(\bar{a}_{0}, \bar{b}\right) \& \neg \theta\left(\bar{a}_{2}, \bar{b}\right)$. Permuting the entries of $\bar{b}$ we can write $\bar{b}=\bar{b}_{0} \bar{b}_{1}$ such that $\bar{b}_{0} \subseteq \bar{a}_{1}$ and $\bar{b}_{1} \cap \bar{a}_{1}=\varnothing$. From (4) there exist $\bar{a}_{0}^{\prime}$ and $\bar{a}_{2}^{\prime}$ such that $\bar{a}_{0}^{\prime} E \bar{a}_{0}, \bar{a}_{2}^{\prime} E \bar{a}_{2}$, and $\left(\bar{a}_{0}^{\prime} \cup \bar{a}_{2}^{\prime}\right) \cap\left(\bar{b} \cup \bar{a}_{1}\right)=\varnothing$. Let $x_{i}$ be an entry of $\bar{x}$. Since $\phi(\bar{x})$ is totally nonalgebraic, $\operatorname{Rk}\left(\phi(\bar{x}) \& x_{i}=c\right)<r$ for all $c \in M$. Therefore we can find $\bar{a}_{i}^{\prime}$ such that $\operatorname{tp}\left(\bar{a}_{1}^{\prime} \mid \bar{b}_{1} \cup \bar{a}_{0}^{\prime} \cup \bar{a}_{2}^{\prime}\right)=\operatorname{tp}\left(\bar{a}_{1} \mid \bar{b}_{1} \cup \bar{a}_{0}^{\prime} \cup \bar{a}_{2}^{\prime}\right)$ and $\bar{a}_{1}^{\prime} \cap\left(\bar{a}_{0} \cup \bar{a}_{2} \cup \bar{b}_{0}\right)=\varnothing$. Let $\bar{b}_{0}^{\prime}$ bear the same relation to $\bar{a}_{1}^{\prime}$ as $\bar{b}_{0}$ to $\bar{a}_{1}$, and $\bar{b}^{\prime}$ denote $\bar{b}_{0}^{\prime} \bar{b}_{1}$. Now $\mathscr{M} \vDash \theta\left(\bar{a}_{0}^{\prime}, \bar{b}\right) \& \neg \theta\left(\bar{a}_{2}^{\prime}, \bar{b}\right)$ since $\bar{a}_{0} E \bar{a}_{0}^{\prime}, \bar{a}_{2} E \bar{a}_{2}^{\prime}$, and $\bar{b} \cap\left(\bar{a}_{0} \cup \bar{a}_{0}^{\prime} \cup \bar{a}_{2} \cup \bar{a}_{2}^{\prime}\right)=\varnothing$. Hence, $\mathscr{M} \vDash \theta\left(\bar{a}_{0}^{\prime}, \bar{b}^{\prime}\right) \& \neg \theta\left(\bar{a}_{2}^{\prime}, \bar{b}^{\prime}\right)$ since $\operatorname{tp}\left(\bar{b}^{\prime} \mid \bar{a}_{0}^{\prime} \cup \bar{a}_{2}^{\prime}\right)=\operatorname{tp}\left(\bar{b} \mid \bar{a}_{0}^{\prime} \cup \bar{a}_{2}^{\prime}\right)$. Since $\bar{a}_{0} E \bar{a}_{0}^{\prime}$ and $\bar{a}_{2} E \bar{a}_{2}^{\prime}$, we have $\mathscr{M} \vDash \theta\left(\bar{a}_{0}, \bar{b}^{\prime}\right) \& \neg \theta\left(\bar{a}_{2}, \bar{b}^{\prime}\right)$ with $\bar{b}^{\prime}$ disjoint from 
each of $\bar{a}_{0}, \bar{a}_{1}, \bar{a}_{2}$. So $\bar{b}^{\prime}$ witnesses that either $\bar{a}_{0} E \bar{a}_{1}$ or $\bar{a}_{1} E \bar{a}_{2}$ fails. This completes the proof that $E$ is an equivalence relation.

Let $\bar{a}_{i}(i \leq m)$ represent distinct $E$-classes. For each pair $(i, j)$ with $i<j \leq m$ there is a basic formula $\theta_{i j}\left(\bar{x}, \bar{b}_{i j}\right)$ such that $\bar{b}_{i j} \cap\left(\bar{a}_{i} \cup \bar{a}_{j}\right)=\varnothing$ and $\mathscr{M} \vDash \theta_{i j}\left(\bar{a}_{i}, \bar{b}_{i j}\right) \& \neg \theta_{i j}\left(\bar{a}_{j}, \bar{b}_{i j}\right)$. Using (4) we obtain $\bar{a}_{i}^{\prime}$ such that $\bar{a}_{i}^{\prime} E \bar{a}_{i}$ and $\bar{a}_{i}^{\prime} \cap \bar{b}_{j k}=\varnothing(i \leq m, j<k \leq m)$. Let $D$ denote $\bigcup\left\{\bar{b}_{j k}: j<k \leq m\right\}$. Now $\bar{a}_{0}^{\prime}, \ldots, \bar{a}_{m}^{\prime}$ realize explicitly contradictory types of rank $r$ over $D$, which contradicts $\operatorname{Mt}(\phi(\bar{x}))=m$. Therefore there are at most $m E$-classes. The argument used for (1) may easily be refined to show that there are at least $m$ $E$-classes. This completes the proof of (5).

(6) Suppose that $\bar{a}_{0} E \bar{a}_{1}$. By (4) choose $\bar{a}$ in the same $E$-class as $\bar{a}_{0}$ and $\bar{a}_{1}$ such that $\bar{a} \cap\left(\bar{a}_{0} \cup \bar{a}_{1}\right)=\varnothing$. Since $\bar{a} E \bar{a}_{0}$, we have $\operatorname{tp}\left(\bar{a} \mid M \backslash \bar{a} \bar{a}_{0}\right)=$ $\operatorname{tp}\left(\bar{a}_{0} \mid M \backslash \bar{a} \bar{a}_{0}\right)$, and so there is an automorphism of $\mathscr{M}$ which switches $\bar{a}$ and $\bar{a}_{0}$ and fixes $M \backslash \bar{a} \bar{a}_{0}$ pointwise. Hence, $\operatorname{Tp}\left(\bar{a}_{0}\right)=\operatorname{Tp}(\bar{a})$. Similarly, $\operatorname{Tp}\left(\bar{a}_{1}\right)=\operatorname{Tp}(\bar{a})$.

We are now ready to prove the lemma. Let $\chi(\bar{x})$ be a $\forall$-formula whose solution set in $\mathscr{M}$ is $I(\mathscr{M})$. From (6) of the previous claim, over $\varnothing$ there are at most $m$ elementary $l(\bar{x})$-types which contain $\chi(\bar{x})$. By Corollary 3.9, in $\mathscr{M}$ the elementary type of a tuple is determined by its $\exists$-type. Clearly, there is a maximal $\forall$-type containing $\chi(\bar{x})$ which is isolated by some $\forall$-formula $\psi(\bar{x})$. Since in $\mathscr{M}$ every solution of $\psi(\bar{x})$ has the same elementary type, $\psi(\bar{x})$ is $T$-complete. Since $\psi(\bar{x})$ implies $\chi(\bar{x})$ and $\chi(\bar{x})$ implies $\phi(\bar{x})$, the proof of the lemma is complete.

In the next lemma we exploit again the idea which gave us the key lemma, Lemma 3.7.

9.4. Lemma. Let $\phi(\bar{x}, \bar{y})$ be a quantifier-free formula such that $\vdash \exists \bar{x} \forall \bar{y} \phi(\bar{x}, \bar{y})$ and let $\bar{y}_{i} \quad(i<\omega)$ be disjoint tuples of variables with $l\left(\bar{y}_{i}\right)=l(\bar{y})$. There exist $k \in \omega$ and a nonnull quantifier-free formula $\psi\left(\bar{y}_{0}, \ldots, \bar{y}_{k}, \bar{z}\right)$ such that, if $\mathscr{N} \vDash T$ and $\bar{b}, \bar{b}_{i}, \bar{c} \subseteq N$, then

$$
\begin{aligned}
\mathscr{N} \vDash \psi\left(\bar{b}_{0}, \ldots, \bar{b}_{k}, \bar{c}\right) & \rightarrow \operatorname{Rk}\left(\neg \phi(\bar{x}, \bar{b}) \& \bigwedge_{i \leq k} \phi\left(\bar{x}, \bar{b}_{i}\right)\right) \\
& <\operatorname{Rk}\left(\bigwedge_{i \leq k} \phi\left(\bar{x}, \bar{b}_{i}\right)\right) .
\end{aligned}
$$

Proof. Let $\mathscr{M}$ be a $\exists \forall$-saturated model of $T$. We study finite sequences $\mathbf{b}=\left\langle\bar{b}_{0}, \ldots, \bar{b}_{k}\right\rangle$ such that $l\left(\bar{b}_{i}\right)=l(\bar{y})$. The set of all such sequences is denoted by $\mathbf{B}$. To establish notation let $B_{i}$ denote the solution set of $\phi\left(\bar{x}, \bar{b}_{i}\right)$, $(i \leq k), r_{i}(\mathbf{b})$ denote $\operatorname{Rk}\left(\left(\bigcap_{j<i} B_{j}\right) \backslash B_{i}\right) \quad(1 \leq i \leq k)$, and $\nu(\mathbf{b})$ denote $\left(r_{1}(\mathbf{b}), \ldots, r_{k}(\mathbf{b})\right)$. The sequence $\mathbf{b}$ is called good if $r_{i}(\mathbf{b}) \geq \operatorname{Rk}\left(\bigcap_{j \leq i} B_{j}\right)$ for all $i, 1 \leq i \leq k$. The range of $\nu$ is ordered lexicographically. Using the same idea as in the proof of Lemma 3.7 we can prove 
Claim. Amongst the good sequences $\mathbf{b}$ is one for which $\nu(\mathbf{b})$ is maximum.

Let $\nu$ be the maximum value of $\nu(\mathbf{b})$ referred to in the Claim. Fix a good sequence $\mathbf{d}=\left\langle\bar{d}_{0}, \ldots, \bar{d}_{k}\right\rangle \in \mathbf{B}$ such that $\nu(\mathbf{d})=\nu$. Let $D_{i}$ denote the solution set of $\phi\left(\bar{x}, \bar{d}_{i}\right)$. Let $r_{i}$ denote $r_{i}(\mathbf{d}) \quad(1 \leq i \leq k)$ and $r$ denote $\operatorname{Rk}\left(\bigcap_{i \leq k} D_{i}\right)$. Notice that $r$ is the same for any $\mathbf{d}$ such that $\nu(\mathbf{d})=\nu$. From Lemma 0.2 there is a set of existential formulas $\Gamma\left(\bar{y}_{0}, \ldots, \bar{y}_{k}\right)$ such that for any sequence $\mathbf{b}=\left\langle\bar{b}_{0}, \ldots, \bar{b}_{k}\right\rangle$ in an $L(T)$-structure $\mathcal{N}, \mathcal{N} \vDash \Gamma\left(\bar{b}_{0}, \ldots, \bar{b}_{k}\right)$ iff $r_{i}(\mathbf{b}) \geq r_{i}(1 \leq i \leq k)$. Similarly, there is a set $\Delta\left(\bar{y}_{0}, \ldots, \bar{y}_{k}, \bar{y}\right)$ of existential formulas such that for any $\mathbf{b}$ and $\bar{b}$ in an $L(T)$-structure $\mathscr{N}, \mathscr{N} \vDash$ $\Delta\left(\bar{b}_{0}, \ldots, \bar{b}_{k}, \bar{b}\right)$ iff $\operatorname{Rk}\left(\left(\bigcap_{i \leq k} B_{i}\right) \backslash B\right) \geq r$, where $B$ denotes the solution set of $\phi(\bar{x}, \bar{b})$. Note that the set of formulas $\Gamma\left(\bar{y}_{0}, \ldots, \bar{y}_{k}\right) \cup \Delta\left(\bar{y}_{0}, \ldots, \bar{y}_{k}, \bar{y}\right)$ is not satisfiable in $\mathscr{M}$, and hence not satisfiable in any model of $T$ since $\mathscr{M}$ is $\exists \forall$-saturated. For, if there are $\bar{b}_{0}, \ldots, \bar{b}_{k}, \bar{b} \subseteq M$ such that $\mathscr{M} \vDash$ $\Gamma\left(\bar{b}_{0}, \ldots, \bar{b}_{k}\right) \cup \Delta\left(\bar{b}_{0}, \ldots, \bar{b}_{k}, \bar{b}\right)$, we can find a good sequence $\mathbf{b}^{\prime} \in B$ such that $\nu\left(\mathbf{b}^{\prime}\right)>\nu$, a contradiction.

By compactness there are $\gamma\left(\bar{y}_{0}, \ldots, \bar{y}_{k}\right)$, the conjunction of a finite subset of $\Gamma\left(\bar{y}_{0}, \ldots, \bar{y}_{k}\right)$, and $\delta\left(\bar{y}_{0}, \ldots, \bar{y}_{k}, \bar{y}\right)$, the conjunction of a finite subset of $\Delta\left(\bar{y}_{0}, \ldots, \bar{y}_{k}, \bar{y}\right)$, such that $\vDash \gamma\left(\bar{y}_{0}, \ldots, \bar{y}_{k}\right) \rightarrow \neg \delta\left(\bar{y}_{0}, \ldots, \bar{y}_{k}, \bar{y}\right)$. Let $\psi\left(\bar{y}_{0}, \ldots, \bar{y}_{k}, \bar{z}\right)$ be a quantifier-free formula such that $\exists \bar{z} \psi\left(\bar{y}_{0}, \ldots, \bar{y}_{k}, \bar{z}\right)$ is equivalent to $\gamma\left(\bar{y}_{0}, \ldots, \bar{y}_{k}\right)$. From above $\mathscr{M} \vDash \Gamma\left(\bar{d}_{0}, \ldots, \bar{d}_{k}\right)$ and so there exists $\bar{e}$ such that $\mathscr{M} \vDash \psi\left(\bar{d}_{0}, \ldots, \bar{d}_{k}, \bar{e}\right)$, whence $\psi\left(\bar{y}_{0}, \ldots, \bar{y}_{k}, \bar{z}\right)$ is nonnull. Further, if $\mathcal{N} \vDash T$ and $\bar{b}_{i}, \bar{c} \subseteq N$ are tuples such that $\mathscr{N} \vDash \psi\left(\bar{b}_{0}, \ldots, \bar{b}_{k}, \bar{c}\right)$, then $\mathscr{N} \vDash \gamma\left(\bar{b}_{0}, \ldots, \bar{b}_{k}\right)$ and so $\mathscr{N} \vDash \forall \bar{y} \neg \delta\left(\bar{b}_{0}, \ldots, \bar{b}_{k}, \bar{y}\right)$. Thus for all $\bar{b} \subseteq N, \mathcal{N} \vDash \Delta\left(\bar{b}_{0}, \ldots, \bar{b}_{k}, \bar{b}\right)$ fails, which means that $\operatorname{Rk}\left(\left(\bigcap_{i<k} B_{i}\right) \backslash B\right)<r$. Towards a contradiction suppose that $\operatorname{Rk}\left(\bigcap_{i<k} B_{i}\right)<r$. By the definability of rank, $\operatorname{Rk}\left(\bigcap_{i \leq k} B_{i}\right)<r$ is implied by some $\forall$-sentence $\chi\left(\bar{b}_{0}, \ldots, \bar{b}_{k}\right)$ true in $\mathscr{N}$. Since $\mathscr{M} \equiv \mathscr{N}$ there must exist $\mathbf{b}^{\prime}=\left\langle\bar{b}_{0}^{\prime}, \ldots, \bar{b}_{k}^{\prime}\right\rangle$ in $\mathscr{M}$ such that $\mathscr{M} \vDash \chi\left(\bar{b}_{0}^{\prime}, \ldots, \bar{b}_{k}^{\prime}\right)$ and hence such that $\operatorname{Rk}\left(\bigcap_{i \leq k} B_{i}^{\prime}\right)<r$, where $B_{i}^{\prime}$ denotes the solution set of $\phi\left(\bar{x}, \bar{b}_{i}^{\prime}\right)$. This contradicts the choice of $\nu$. Therefore, $\operatorname{Rk}\left(\bigcap_{i \leq k} B_{i}\right) \geq r$ and so $\psi\left(\bar{y}_{0}, \ldots, \bar{y}_{k}, \bar{z}\right)$ has the desired properties.

9.5. Lemma. Let $\phi(\bar{x}, \bar{y})$ be a quantifier-free formula. There is a quantifierfree formula $\phi^{*}(\bar{x}, \bar{y})$ such that

$$
\vdash\left[\forall \bar{y} \phi(\bar{x}, \bar{y}) \rightarrow \forall \bar{y} \phi^{*}(\bar{x}, \bar{y})\right] \&\left[\forall \bar{y}\left[\bar{y} \cap \bar{x}=\varnothing \rightarrow \phi^{*}(\bar{x}, \bar{y})\right] \rightarrow \forall \bar{y} \phi(\bar{x}, \bar{y})\right] .
$$

Since it is easy we omit the proof of Lemma 9.5. The next lemma provides the tool we need to build prime models.

9.6. Lemma. If $\phi(\bar{x}, \bar{y})$ is a quantifier-free formula and $\vdash \exists \bar{x} \forall \bar{y} \phi(\bar{x}, \bar{y})$, then there is a T-complete $\forall$-formula $\psi(\bar{x}, \bar{u})$ which implies $\forall \bar{y} \phi(\bar{x}, \bar{y})$.

Proof. From Lemma 9.5 we can suppose that $\forall \bar{y}[\bar{y} \cap \bar{x}=\varnothing \rightarrow \phi(\bar{x}, \bar{y})]$ implies $\forall \bar{y} \phi(\bar{x}, \bar{y})$. Let $\psi\left(\bar{y}_{0}, \ldots, \bar{y}_{k}, \bar{z}\right)$ be a nonnull quantifier-free formula satisfying the conclusion of Lemma 9.4. From Lemma 9.3 there is a $T$-complete 
$\forall$-formula $\chi=\chi\left(\bar{y}_{0}, \ldots, \bar{y}_{k}, \bar{z}\right)$ which implies $\psi\left(\bar{y}_{0}, \ldots, \bar{y}_{k}, \bar{z}\right)$. Let $T^{\prime}=$ $T[\chi]$ and $\rho=\rho\left(\bar{y}_{0}, \ldots, \bar{y}_{k}, \bar{z}\right)$ be the conjunction of all the atomic formulas $R(u)$, where $u$ is an entry of $\bar{y}_{0}, \cdots \bar{y}_{k} \bar{z}$ and $R$ is the corresponding unary relation introduced in the formation of $L\left(T^{\prime}\right)$. Let $\phi^{\prime}(\bar{x})$ denote the formula

$$
\exists \bar{y}_{0} \cdots \exists \bar{y}_{k} \exists \bar{z}\left[\rho\left(\bar{y}_{0}, \ldots, \bar{y}_{k}, \bar{z}\right) \& \bigwedge_{i \leq k} \phi\left(\bar{x}, \bar{y}_{i}\right)\right]
$$

and $r$ denote $\operatorname{Rk}\left(\phi^{\prime}(\bar{x})\right)$. Note that $\phi^{\prime}(\bar{x})$ is almost quantifier-free in $T^{\prime}$.

Let $\mathscr{N} \vDash T, \bar{b}_{0} \ldots \bar{b}_{k} \bar{c}$ be a solution of $\chi\left(\bar{y}_{0}, \ldots, \bar{y}_{k}, \bar{z}\right)$ in $\mathscr{N}$, and $\mathscr{N}^{\prime}=$ $\mathscr{N}\left[\bar{b}_{0} \cdots \bar{b}_{k} \bar{c}\right]$ be the corresponding model of $T^{\prime}$. Note that

$$
\mathscr{N}^{\prime} \vDash \forall \bar{x}\left[\phi^{\prime}(\bar{x}) \leftrightarrow \bigwedge_{i \leq k} \phi\left(\bar{x}, \bar{b}_{i}\right)\right] .
$$

Since $\mathcal{N} \vDash \psi\left(\bar{b}_{0}, \ldots, \bar{b}_{k}, \bar{c}\right)$, for any $\bar{b} \subseteq N$

$$
\begin{aligned}
\operatorname{Rk}\left(\neg \phi(\bar{x}, \bar{b}) \& \phi^{\prime}(\bar{x})\right) & =\operatorname{Rk}\left(\neg \phi(\bar{x}, \bar{b}) \& \bigwedge_{i \leq k} \phi\left(\bar{x}, \bar{b}_{i}\right)\right) \\
& <\operatorname{Rk}\left(\bigwedge_{i \leq k} \phi\left(\bar{x}, \bar{b}_{i}\right)\right)=\operatorname{Rk}\left(\phi^{\prime}(\bar{x})\right) .
\end{aligned}
$$

By Lemma 9.2 let $\psi^{\prime}(\bar{x})$ be a nonnull $\forall$-formula of $T^{\prime}$ such that for every $\mathscr{M}^{\prime} \vDash T^{\prime}$ and $\bar{a} \subseteq M^{\prime}$

$$
\mathscr{M}^{\prime} \vDash \psi^{\prime}(\bar{a}) \leftrightarrow\left[\phi^{\prime}(\bar{a}) \& \operatorname{Rk}\left(\operatorname{tp}\left(\bar{a} \mid M^{\prime} \backslash \bar{a}\right)\right)=r\right] .
$$

Fix $\bar{a} \subseteq N$ such that $\mathscr{N}^{\prime} \vDash \psi^{\prime}(\bar{a})$. Then $\mathscr{N}^{\prime} \vDash \phi^{\prime}(\bar{a})$ and $\operatorname{Rk}(\operatorname{tp}(\bar{a} \mid N \backslash \bar{a}))=r$. Consider $\bar{b} \subseteq N \backslash \bar{a}$. Since $\operatorname{Rk}\left(\neg \phi(\bar{x}, \bar{b}) \& \phi^{\prime}(\bar{x})\right)<r$ and $\mathscr{N}^{\prime} \vDash \phi^{\prime}(\bar{a})$, we have $\mathscr{N} \vDash \phi(\bar{a}, \bar{b})$. Therefore, $\forall \bar{y}[\bar{y} \cap \bar{a}=\varnothing \rightarrow \phi(\bar{a}, \bar{y})]$, and hence also $\forall \bar{y} \phi(\bar{a}, \bar{y})$, are true in $\mathscr{N}$. Thus $\vDash_{T^{\prime}} \psi^{\prime}(\bar{x}) \rightarrow \forall \bar{y} \phi(\bar{x}, \bar{y})$.

Notice that $\psi^{\prime}(\bar{a})$ says that $\bar{a}$ is independent with respect to $\phi^{\prime}(\bar{x})$ in the sense of the proof of Lemma 9.3. Repeating the final part of the argument for Lemma 9.3 we see that there is a $\forall$-formula $\psi^{\prime \prime}(\bar{x})$ of $T^{\prime}$ which is $T^{\prime}$-complete and implies $\psi^{\prime}(\bar{x})$. To finish we take for $\psi(\bar{x}, \bar{u})$ a $\forall$-formula equivalent to $\left(\psi^{\prime \prime}\right)_{T}\left(\bar{y}_{0}, \ldots, \bar{y}_{k}, \bar{z}, \bar{x}\right)$. That $\psi(\bar{x}, \bar{u})$ is suitable follows from the discussion immediately preceding Lemma 9.3

9.7. Theorem. Let $T$ be a complete $\exists \forall$-theory over a finite relational language. $T$ has a prime model.

Proof. Let $\phi_{i}\left(\bar{x}_{i}, \bar{y}_{i}\right) \quad(i<\omega)$ be quantifier-free formulas such that $\left\{\exists \bar{x}_{i} \forall \bar{y}_{i}\right.$ $\left.\phi_{i}\left(\bar{x}, \bar{y}_{i}\right): i<\omega\right\}$ is an axiomatization of $T$. By induction on $i$ we will find $T$-complete universal formulas $\theta_{i}\left(\bar{x}_{0}, \ldots, \bar{x}_{i}, \bar{z}_{0}, \ldots, \bar{z}_{i}\right)(i<\omega)$ such that for all $i<\omega$

$$
\vDash \theta_{i}\left(\bar{x}_{0}, \ldots, \bar{x}_{i}, \bar{z}_{0}, \ldots, \bar{z}_{i}\right) \rightarrow \forall \bar{y}_{j} \phi_{j}\left(\bar{x}_{j}, \bar{y}_{j}\right)
$$


and

$$
\vDash \theta_{i+1}\left(\bar{x}_{0}, \ldots, \bar{x}_{i+1}, \bar{z}_{0}, \ldots, \bar{z}_{i+1}\right) \rightarrow \theta_{i}\left(\bar{x}_{0}, \ldots, \bar{x}_{i}, \bar{z}_{0}, \ldots, \bar{z}_{i}\right) .
$$

$\theta_{0}\left(\bar{x}_{0}, \bar{z}_{0}\right)$ is obtained by applying Lemma 9.6 to $\phi_{0}\left(\bar{x}_{0}, \bar{y}_{0}\right)$.

Suppose an appropriate $\theta_{i}=\theta_{i}\left(\bar{x}_{0}, \ldots, \bar{x}_{i}, \bar{z}_{0}, \ldots, \bar{z}_{i}\right)$ has been found. Let $T^{\prime}=T\left[\theta_{i}\right]$. As pointed out above $T^{\prime}$ is a complete $\exists \forall$-theory. Applying Lemma 9.6 there is a $\forall$-formula $\psi^{\prime}=\psi^{\prime}\left(\bar{x}_{i+1}, \bar{z}_{i+1}\right)$ complete in $T^{\prime}$ such that

$$
\vDash_{T^{\prime}} \psi^{\prime}\left(\bar{x}_{i+1}, \bar{z}_{i+1}\right) \rightarrow \forall \bar{y}_{i+1} \phi_{i+1}\left(\bar{x}_{i+1}, \bar{y}_{i+1}\right)
$$

Without loss of generality none of the entries of $\bar{x}_{0}, \ldots, \bar{x}_{i} \bar{z}_{0} \ldots \bar{z}_{i}$ occurs in $\psi^{\prime}$. Let $\theta_{i+1}=\theta_{i+1}\left(\bar{x}_{0}, \ldots, \bar{x}_{i+1}, \bar{z}_{0}, \ldots, \bar{z}_{i+1}\right)$ be a $\forall$-formula equivalent to $\left(\psi^{\prime}\right)_{T}$. Now $\theta_{i+1}$ is $T$-complete and $\theta_{i+1}$ implies $\forall \bar{y}_{i+1} \phi_{i+1}\left(\bar{x}_{i+1}, \bar{y}_{i+1}\right)$ since $\psi^{\prime}$ does. Finally, $\theta_{i+1}$ implies $\theta_{i}$ since $\theta_{i}$ is a conjunct of $\left(\psi^{\prime}\right)_{T}$. It is clear that $\theta_{i+1}$ has the desired properties and so the induction is complete.

Let $\mathscr{M} \vDash T$. Choose $\bar{a}_{i}, \bar{c}_{i} \subseteq M \quad(i<\omega)$ such that

$$
\mathscr{M} \vDash \theta_{i}\left(\bar{a}_{0}, \ldots, \bar{a}_{i}, \bar{c}_{0}, \ldots, \bar{c}_{i}\right) \text { for all } i<\omega .
$$

Let $\mathscr{N} \subseteq \mathscr{M}$ be the structure with universe $N=\bigcup\left\{\bar{a}_{i} \bar{c}_{i}: i<\omega\right\}$. Since $\mathscr{M} \vDash \forall \bar{y}_{i} \phi_{i}\left(\bar{a}_{i}, \bar{y}_{i}\right)$, we have $\mathscr{N} \vDash \forall \bar{y}_{i} \phi_{i}\left(\bar{a}_{i}, \bar{y}_{i}\right) \quad(i<\omega)$. Hence $\mathscr{N} \vDash T$. Also, since $\theta_{i}$ is universal, we have $\mathscr{N} \vDash \theta_{i}\left(\bar{a}_{0}, \ldots, \bar{a}_{i}, \bar{c}_{0}, \ldots, \bar{c}_{i}\right)$ for all $i<\omega$. Since the formula $\theta_{i}$ is $T$-complete, $\bar{a}_{0} \cdots \bar{a}_{i} \bar{c}_{0} \cdots \bar{c}_{i}$ realizes an isolated type in $\mathscr{N}$. Therefore, every tuple $\bar{b} \subseteq N$ realizes an isolated type in $\mathscr{N}$. Clearly $\mathscr{N}$ is a prime model of $T$.

\section{ConClusion}

In this paper and its predecessor we have collected a substantial body of information about complete coinductive theories. However, there are many aspects of the subject which remain obscure. We hope to stimulate further interest by listing some conjectures.

Recall the notion of independence based on $\exists: \forall$-formulas developed in $\S 7$. The negation of independence gives us a forking notion based on $\exists: \forall$-formulas. By analogy with the usual forking notion, if $B \subseteq C$ and $\exists: \forall-\operatorname{tp}(\bar{a} \mid C)$ forks over $B$, then the type forks via some $\exists: \forall$-formula over $C$. In this context we have

10.1. Conjecture. Every type which forks forks via some $\exists$-formula.

A question we have left wholly unexplored is the relation between forking with respect to $\exists: \forall$-formulas and forking with respect to all formulas. It appears that, if $\bar{a}, \bar{b}, C \subseteq M$ and $\mathscr{M}$ is a model of a complete coinductive theory $\exists \forall$-saturated over $\bar{C}$, then $\bar{a} \downarrow \bar{b} \quad(C)$ means the same whether we work with all formulas or just $\exists: \forall$-formulas.

There are some interesting questions about prime models. To avoid trivial counterexamples for the rest of the paper we confine consideration to finite relational languages. 
10.2. Conjecture. Let $\mathscr{M}$ be a model of a complete coinductive theory which is $\exists \forall$-saturated over $A \subseteq M$. Then there exists a prime model over $A$.

From Lemma 7.7 there is an equivalence relation $E_{\varnothing}$ defined by the (not necessarily first-order) formula $x=y \vee \neg\left[\begin{array}{lll}x \downarrow y & (\varnothing)\end{array}\right]$. The $E_{\varnothing}$-classes are called the components of a model. It is not hard to show that in the prime model of Theorem 9.7 all components are finite.

10.3. Conjecture. If $\mathscr{M}$ is a countable model of a complete coinductive theory in which all components are finite, then $\mathscr{M}$ is a prime model.

10.4. Conjecture. If $\operatorname{Th}(\mathscr{M})$ is coinductive and all the components of $\mathscr{M}$ are finite, then $\mathscr{M}$ is $\aleph_{0}$-categorical.

Before stating our final conjecture we need some notation and a lemma. Let $\varphi=\varphi(x)$ be a formula with one free variable which has a solution in the structure $\mathscr{M}$. By $\mathscr{M}_{\varphi}$ we denote the substructure of $\mathscr{M}$ whose universe is the solution set of $\varphi$ in $\mathscr{M}$.

10.5. Lemma. Let $\operatorname{Th}(\mathscr{M})$ be coinductive and $\varphi=\varphi(x)$ be a $\forall$-formula which has a solution in $\mathscr{M}$. Then $\operatorname{Th}\left(\mathscr{M}_{\varphi}\right)$ is also coinductive.

Proof. Note that $T_{\varphi}=\operatorname{Th}\left(\mathscr{M}_{\varphi}\right)$ is determined by $T=\operatorname{Th}(\mathscr{M})$. From the Claim used in the proof of Lemma 0.1 it is sufficient to find a model $\mathscr{N}$ of $T_{\varphi}$ such that for any $\mathscr{N}^{\prime} \succeq \mathscr{N}$ every structure lying between $\mathscr{N}$ and $\mathscr{N}^{\prime}$ is also a model of $T_{\varphi}$. To this end let $\mathscr{N}$ be a saturated model of $T_{\varphi}$, of cardinality $\lambda$ say. Without loss of generality we can suppose that $\mathscr{N}^{\prime} \succeq \mathscr{N}$ is saturated of cardinality $\mu>\lambda$. We can choose the cardinals $\lambda$ and $\mu$ such that $T$ has saturated models in both powers. Let $\mathscr{M}$ be saturated of power $\lambda$. Then $\mathscr{M}_{\varphi}$ is also saturated of power $\lambda$. Thus we may suppose that $\mathscr{N}=\mathscr{M}_{\varphi}$. Since up to automorphisms of the large model a saturated model of power $\lambda$ can sit in only one way in a saturated model of power $\mu$, there exists $\mathscr{M}^{\prime} \succeq \mathscr{M}$ such that $\mathscr{M}^{\prime}$ is saturated of power $\mu$ and $\left(\mathscr{M}^{\prime}\right)_{\varphi}=\mathscr{N}^{\prime}$. Consider $\mathscr{N}^{*}$ such that $\mathscr{N} \subseteq \mathscr{N}^{*} \subseteq \mathscr{N}^{\prime}$, and let $\mathscr{M}^{*}$ denote $\mathscr{M} \cup N^{*}$. Since $T$ is coinductive, $\mathscr{M}^{*} \vDash T$. the members of $N^{*}$ satisfy $\varphi(x)$ in $\mathscr{M}^{*}$ since they satisfy $\varphi(x)$ in $\mathscr{N}^{\prime}$. The members of $M^{*} \backslash N^{*}=M \backslash N$ satisfy $\neg \varphi(x)$ in $\mathscr{M}^{*}$ since they satisfy $\neg \varphi(x)$ in $\mathscr{M}$. Therefore, $\mathscr{N}^{*}=\left(\mathscr{M}^{*}\right)_{\varphi} \Vdash T$ which completes the proof of the lemma.

10.6. Conjecture. Let $\mathscr{M}$ be a prime model of a complete coinductive theory. There exist $\forall$-formulas $\varphi_{i}(\chi) \quad(i<\omega)$ such that $\varphi_{i}$ implies $\varphi_{i+1}, \mathscr{M}_{\varphi_{i}}$ is $\aleph_{0}$ categorical $(i<\omega)$, and $M=\bigcup\left\{\mathscr{M}_{\varphi_{i}}: i<\omega\right\}$.

If this last conjecture were true, it would yield a clear picture of the prime model. Let $T$ be the first example of a complete coinductive theory found in the introduction to Part I of this paper, where the prime model is a countable disjoint union of finite graphs without cycles. Notice that in this case there are two quite different ways of approximating the prime model. We can take $\varphi_{i}(x)$ 
to mean " $x$ belongs to a component of size $\leq i+1$ " or to mean " $x$ belongs to a component of diameter $\leq i$ ". The latter meaning does not satisfy the conclusion of the last conjecture because $\mathscr{M}_{\varphi_{i}}$ has arbitrarily large components. However, it yields what in some sense is a better approximation. Thus, it may well be fruitful to consider approximations to the prime model via $\forall$-formulas other than those contemplated in the conjecture.

\section{REFERENCES}

1. J. T. Baldwin, Definable second-order quantifiers, Model-Theoretic Logics, Springer-Verlag, New York, 1985, pp. 445-477.

2. J. T. Baldwin and D. W. Kueker, Ramsey quantifiers and the finite cover property, Pacific J. Math. 90 (1980), 11-19.

3. J. T. Baldwin and S. Shelah, Second-order quantifiers and the complexity of theories, Notre Dame J. Formal Logic 26 (1985), 229-303.

4. G. Cherlin and A. H. Lachlan, Finitely homogeneous structures, Trans. Amer. Math. Soc. 296 (1986), 815-850.

5. V. Harnik and L. Harrington, Fundamentals of forking, Ann. Pure Appl. Logic 26 (1984), 245-286.

6. I. M. Hodkinson and H. D. Macpherson, Relational structures induced by their finite induced substructures, J. Symbolic Logic 53 (1988), 222-230.

7. E. Hrushovski, Remarks on $\aleph_{0}$-stable $\aleph_{0}$-categorical theories, preprint.

8. A. H. Lachlan, Two conjectures on the stability of $\omega$-categorical theories, Fund. Math. 81 (1974), 133-145.

9. _ Complete theories with only universal and existential axioms, J. Symbolic Logic 52 (1987), 698-711.

10. _ Complete coinductive theories. I, Trans. Amer. Math. Soc. 319 (1990), 209-241.

11. _ Some coinductive graphs, Arch. Math. Logik 29 (1990), 213-229.

12. H. D. Macpherson, Graphs determined by their finite induced subgraphs, J. Combin. Theory Ser. B 41 (1986), 230-234.

13. J. Schmerl, Coinductive $\aleph_{0}$-categorical theories, J. Symbolic Logic 55 (1990), 1130-1137.

14. S. Shelah, Classification theory and the number of nonisomorphic models, North-Holland, Amsterdam, 1978.

Department of Mathematics and Statistics, Simon Fraser University, Burnaby, British Columbia V5A 1S6, Canada 